\title{
An uncertainty model of acoustic metamaterials with random parameters
}

\author{
Z. C. $\mathrm{He}^{\mathrm{a}}$, J. Y. $\mathrm{Hu}^{\mathrm{a}}$, Eric Li ${ }^{\text {b,* }}$ \\ ${ }^{a}$ State Key Laboratory of Advanced Design and Manufacturing for Vehicle Body, Hunan University, Changsha, \\ 410082 P. R. China \\ ${ }^{\mathrm{b}}$ School of Science, Engineering \& Design, Teesside University, Middlesbrough, UK
}

\begin{abstract}
Acoustic metamaterials (AMs) are man-made composite materials. However, the random uncertainties are unavoidable in the application of AMs due to manufacturing and material errors which lead to the variance of the physical responses of AMs. In this paper, an uncertainty model based on the change of variable perturbation stochastic finite element method (CVPS-FEM) is formulated to predict the probability density functions of physical responses of AMs with random parameters. Three types of physical responses including the band structure, mode shapes and frequency response function of AMs are studied in the uncertainty model, which is of great interest in the design of AMs. In this computation, the physical responses of stochastic AMs are expressed as linear functions of the pre-defined random parameters by using the first-order Taylor series expansion and perturbation technique. Then, based on the linear function relationships of parameters and responses, the probability density functions of the responses can be calculated by the change-of-variable technique. Three numerical examples are employed to demonstrate the effectiveness of the CVPS-FEM for stochastic AMs, and the results are validated by Monte Carlo method (MCM) successfully.
\end{abstract}

Key words: Acoustic metamaterials; Random parameters; Perturbation stochastic finite element method; Change-of-variable technique; Probability density function.

\footnotetext{
* Corresponding author; Eric Li (ericsg2012@gmail.com)
} 


\section{Introduction}

Recently, acoustic metamaterials (AMs) have attracted much attention among the researchers due to their unique physical characteristics [1-5]. The useful properties of AMs depend on their periodic microstructures, instead of the chemical reactions during manufacturing [6], and AMs have been widely used to solve the acoustic problems [7-9]. For example, elastic wave propagating in AMs can be stopped or attenuated when their frequencies are within or close to the band gaps [10]. In parallel with theoretical studies, AMs have been applied in many fields, such as structural vibration mitigation [11], invisible acoustic cloaking [12] and acoustic waveguides [13]. In the early study of AMs, band gaps are based on Bragg scattering effect. It is noted that Bragg band gaps usually exist in the frequency region where the wavelength of the waves is in the same order of magnitude with the lattice constant, which limits the practical application of AMs in low-frequency region [14].

To overcome the limitation of Bragg scattering effect, another type of AMs called locally resonant acoustic metamaterials (LRAMs) have become a hot research topic in recent years. The LRAMs made up of rubber-coated lead balls was first proposed by Liu et al. in 2000, and the size of the structural unit is two orders of magnitude smaller than the wavelength [15]. The band gaps in LRAMs are due to the local resonance of the scatterers, hence the dimension of AMs can be smaller than the wavelength [10]. Based on locally resonant mechanism, LRAMs have been applied to solve the low-frequency noise and vibration problems. Sheng et al. [16] proposed a three-component LRAMs, which gave the 
band gap at 350-2000Hz. The band gaps and vibrations in 1-D and 2-D mass-spring structures were extensively studied by Jensen et al. [17]. Later, the band gaps and negative effective mass of a 1-D mass-spring system were experimentally demonstrated by Yao et al. [18]. Recently, based on locally resonant mechanism, Lu et al. [1] proposed a topology optimization method for the optimal design of LRAMs to obtain negative bulk modulus at certain frequency ranges.

An efficient numerical algorithm for the simulation of AMs is of great importance in practical application of AMs. Recently, some computational techniques have been presented to simulate the wave propagation in AMs, such as the plane-wave expansion (PWE) method [19] and the finite-difference time-domain (FDTD) method [20]. PWE method is one of the most widely used techniques for the calculation of AMs, however, it has an inherent drawback of poor convergence [21]. Furthermore, it is difficult for FDTD to simulate AMs with complex geometries because of the staircase effect [22]. Recently, finite element method (FEM) has become a popular method to simulate AMs. Based on the frame of FEM, lumped mass (LM) method was proposed to calculate the wave propagation in phononic crystals by Wang et al. [23]. In addition, the finite element time-domain method was developed to analyze the optical black hole in metamaterials by Yang et al. [24]. A mixed finite element methods was proposed to analyze the wave propagation in double negative metamaterials by Li [25]. Consequently, smoothed finite element method (S-FEM) has been applied to compute the band gap of AMs [26-29].

It is well known that the manufacturing tolerances, unpredictable environment factors, and the material properties errors always exist, leading to the variance of the physical 
response of AMs. Therefore, in the design and application of AMs, it is of great significance to propose an efficient uncertainty model to predict the physical responses of AMs with consideration of uncertainty effects. Generally, the uncertainties of engineering structural parameters are defined as random variables whose probability density functions can be obtained on the basis of sample data statistical analysis. Recently, the sources of uncertainty with functions of space or time combined with machine learning algorithm have been studied [30, 31].

In general, the uncertain problems with random parameters can be treated as stochastic problems, and a lot of probabilistic approaches have been widely used to solve the these problems, such as the Monte Carlo method (MCM) [32, 33], the spectral stochastic finite element method (SSFEM) $[34,35]$ and the perturbation stochastic finite element method (PSFEM) [36, 37]. Among these probabilistic approaches, the PSFEM has become more and more popular in the analysis of stochastic problems [36]. However, the random results obtained by PSFEM are given in the form of mean value and standard deviations, and the probability density functions of results cannot be obtained unless the results are treated as Gaussian random fields [38]. In order to obtain the probability density functions of the random results when non-Gaussian variables exist, the change of variable perturbation stochastic finite element method (CVPS-FEM) has been proposed by Xia et al. [39] to calculate the probability density functions of responses of stochastic structures. However, the formulations of CVPS-FEM to solve the probability distributions of natural frequencies and mode shapes of stochastic structures are still not available.

To obtain the reliable performance of the AMs with random parameters, the physical 
responses of stochastic AMs including band structure, mode shapes and frequency response function must be predicted accurately. This is extremely important in the design and application of AMs in engineering practices. In order to predict the probability density functions of band structure, mode shapes and frequency response function of AMs with random parameters, the CVPS-FEM is applied to formulate the random uncertainty model of stochastic AMs in this work. In this random uncertainty model, the random physical responses of AMs are approximated as linear functions of the random parameters by using the first-order Taylor series expansion and perturbation technique, then, based on the linear function relationships of parameters and responses, the probability density functions of the responses can be calculated by the change-of-variable technique.

The remainder of this paper is organized as follows. In Section 2, the calculation of the probability density functions for linear functions by using change-of-variable technique is presented, and the elastic wave in AMs is discussed. The formulation of the uncertainty model by using the CVPS-FEM to predict the random band structure, mode shapes and frequency response function of stochastic AMs is illustrated in Section 3. Three numerical examples are used to demonstrate the accuracy of the uncertain model of AMs in Section 4. Several conclusions are given in Section 5.

\section{Mathematical backgrounds}

\subsection{Change-of-variable technique for linear functions}

Assume that $g=h\left(x_{1}, \ldots, x_{m}\right)$ is a linear function with $m$ independent random parameters, which is defined as 


$$
g=h\left(x_{1}, \ldots, x_{m}\right)=A_{0}+\sum_{i=1}^{m} A_{i} x_{i}, \quad i=1, \ldots, m
$$

where the probability density function of $x_{i}$ can be given as $p_{x_{i}}\left(x_{i}\right)$.

Based on change-of-variable technique [39], the probability density function of $g$ can be given as

$$
p_{g}(g)= \begin{cases}\frac{1}{\left|A_{1}\right|} p_{x_{1}}\left(\frac{g-A_{0}}{A_{1}}\right) & (m=1) \\ \frac{1}{\left|A_{2}\right|} \int_{x_{1}=-\infty}^{\infty} p_{x_{1}}\left(x_{1}\right) p_{x_{2}}\left(\frac{g-A_{0}-A_{1} x_{1}}{A_{2}}\right) \mathrm{d} x_{1} & (m=2) \\ \int_{y_{m}=-\infty}^{\infty} p_{y_{m}}\left(y_{m}\right) p_{Y_{m-2}}\left(g-A_{0}-A_{m} y_{m}\right) \mathrm{d} y_{m} & (m>2)\end{cases}
$$

where $y_{\mathrm{m}}=A_{m} x_{m}, \quad Y_{m-2}=\sum_{i=1}^{m-1} A_{i} x_{i}$.

\subsection{Elastic wave in AMs}

Assume that the media where the elastic wave propagates is isotropic, and the governing equation of the elastic wave in the frequency domain can be given as

$$
(\lambda+\mu) \nabla(\nabla \cdot \mathbf{u}(\mathbf{r}))+\mu \nabla^{2} \mathbf{u}(\mathbf{r})=-\rho \omega^{2} \mathbf{u}(\mathbf{r})
$$

where $\mathbf{r}=(x, y, z)$ is the position vector, $\mathbf{u}(\mathbf{r})$ is the displacement vector, $\nabla=(\partial / \partial x, \partial / \partial y, \partial / \partial z)$ is the vector differential operator and $\omega$ is the angular frequency of the propagating wave. $\lambda$ and $\mu$ are Lamé's first parameter and shear modulus, respectively, which can be given as

$$
\lambda=\frac{v E}{(1+v)(1-2 v)}, \quad \mu=\frac{E}{2(1+v)}
$$

where $E$ is the Young's Modulus, and $v$ is the Poisson's ratio.

Based on the Bloch's theorem of wave propagation in the periodic structure, the 
displacement vector $\mathbf{u}(\mathbf{r})$ for the two-dimensional periodic AMs can be expressed as

$$
\mathbf{u}(\mathbf{r})=e^{i(\mathbf{k}-\mathbf{r})} \mathbf{u}_{\mathbf{k}}(\mathbf{r})
$$

where $\mathbf{u}_{\mathbf{k}}(\mathbf{r})$ is the periodic function which has the same periodic characteristic with the AMs, the wave vector $\mathbf{k}=\left(k_{x}, k_{y}\right)$ is limited in the first Brillouin zone, and $\mathbf{r}=\mathbf{r}(x, y)$ is the position vector.

By using the Bloch's theorem, the solution of the periodic AMs structure system can be reduced one unit cell. Then, based on the finite element method, Eq. (3) of the unit cell can be expressed as

$$
\left[\mathbf{K}-\omega^{2} \mathbf{M}\right] \mathbf{U}=0
$$

where $\mathbf{K}$ and $\mathbf{M}$ are the stiffness and mass matrices, respectively, and $\mathbf{U}$ is the nodal displacement.

By virtue of the periodic Bloch boundary conditions [40], the nodal displacement $\mathbf{U}(\mathbf{r})$ on the boundary will meet the following equation:

$$
\mathbf{U}(\mathbf{r}+\mathbf{a})=e^{i(\mathbf{k}+\mathbf{a})} \mathbf{U}(\mathbf{r})
$$

where $\mathbf{a}$ is the lattice constant of periodic AMs structure.

Combined with Eq. (7), Eq. (6) can be calculated for any wave vector $\mathbf{k}$ limited in the first Brillouin zone. Furthermore, this area is limited in the irreducible Brillouin zone (IBZ) for the square and hexagon lattice as plotted in Fig. 1. The band structure can be obtained when the wave vector $\mathbf{k}$ is adopted along different directions of $\mathrm{M} \rightarrow \Gamma \rightarrow \mathrm{X} \rightarrow \mathrm{M}$ on the boundary of the IBZ. The band gap exists along the $\omega$ axis when two adjacent dispersion curves do not overlap. 

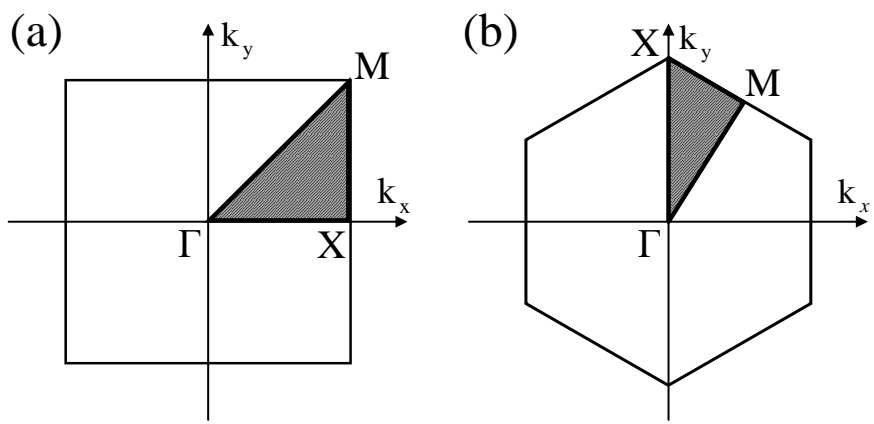

Fig. 1. (a) The first Brillouin zone (square) and the irreducible Brillouin zone (triangle) for a square lattice; (b)

The first Brillouin zone (hexagon) and the irreducible Brillouin zone (triangle) for a hexagon lattice.

\section{CVPS-FEM for AMs with random parameters}

In this work, all uncertain parameters existing in AMs are described as random parameters, which belong to a random vector $\mathbf{a}=\left(a_{1}, a_{2}, \ldots, a_{m}\right)$, and the probability density functions of these random parameters are given as $\mathbf{p}_{\mathbf{a}}(\mathbf{a})=\left(p_{a_{1}}\left(a_{1}\right), p_{a_{2}}\left(a_{2}\right), \ldots, p_{a_{m}}\left(a_{m}\right)\right)$.

As mentioned in Section 2, based on FEM and periodic Bloch conditions, the band structure and mode shapes of AMs can be calculated. Furthermore, the amplitude of frequency response of AMs is sharply reduced at the frequency range where the band gaps exist, hence the band gaps can be verified by calculating the frequency response function. However, when the uncertain random parameters are introduced, these physical responses of AMs mentioned above are changed. In this section, the CVPS-FEM is formulated, and the probability distributions of the random physical responses including the band structure, mode shapes and frequency response function of AMs are calculated.

\subsection{CVPS-FEM for band structure of AMs with random parameters}

By introducing the periodic Bloch boundary conditions, for each wave vector $\mathbf{k}$ on the boundary of the IBZ, Eq. (6) for the unit cell can be rewritten as 


$$
\mathbf{K} \mathbf{U}_{j}=\omega_{j}^{2} \mathbf{M} \mathbf{U}_{j}, \quad \mathbf{j}=1,2, \ldots, n
$$

The normalization condition can be expressed as

$$
\mathbf{U}_{j}^{T} \mathbf{M} \mathbf{U}_{j}=1
$$

It has been proven [41] that the mass matrix $\mathbf{M}$ is always symmetric positive definite because the shape functions satisfy the partitions of unity property, and Eq. (9) will be satisfied based on the so-called Rayleigh Quotient [42].

Considering the existence of the random parameters, Eq. (8) can be expressed as

$$
\mathbf{K}(\mathbf{a}) \mathbf{U}_{j}(\mathbf{a})=\omega_{j}^{2}(\mathbf{a}) \mathbf{M}(\mathbf{a}) \mathbf{U}_{j}(\mathbf{a})
$$

where $\mathbf{K}(\mathbf{a}), \mathbf{M}(\mathbf{a}), \omega_{j}(\mathbf{a})$ and $\mathbf{U}_{j}(\mathbf{a})$ are the stiffness matrix, mass matrix, $j^{\text {th }}$ natural angular frequency and $j^{\text {th }}$ mode shape of the AMs with considering random parameters, respectively.

The first-order Taylor series expansion for the linear function is exact, and the accuracy of it for the non-linear function is also acceptable if the deviation of uncertain parameter is small [43]. On the other hand, by using the higher order Taylor expansion with expensive computational cost, the improvement of accuracy is rather small [39]. As our main focus in this work is to study the small deviation of random parameters of AMs, only the first-order Taylor series expansion is used.

Based on the first-order Taylor series expansion, the stiffness matrix $\mathbf{K}(\mathbf{a})$ and mass matrix $\mathbf{M}(\mathbf{a})$ can be expressed as

$$
\mathbf{K}(\mathbf{a})=\mathbf{K}\left(\mathbf{a}^{e}\right)+\sum_{i=1}^{m} \frac{\partial \mathbf{K}\left(\mathbf{a}^{e}\right)}{\partial a_{i}}\left(a_{i}-a_{i}^{e}\right)=\mathbf{K}^{e}+\Delta \mathbf{K}
$$




$$
\mathbf{M}(\mathbf{a})=\mathbf{M}\left(\mathbf{a}^{e}\right)+\sum_{i=1}^{m} \frac{\partial \mathbf{M}\left(\mathbf{a}^{e}\right)}{\partial a_{i}}\left(a_{i}-a_{i}^{e}\right)=\mathbf{M}^{e}+\Delta \mathbf{M}
$$

where $\mathbf{a}^{e}$ is the mean value of $\mathbf{a}=\left(a_{1}, a_{2}, \ldots, a_{m}\right), \mathbf{K}^{e}$ and $\Delta \mathbf{K}$ are the mean value and deviation value of $\mathbf{K}(\mathbf{a}), \mathbf{M}^{e}$ and $\Delta \mathbf{M}$ are the mean value and deviation value of $\mathbf{M}(\mathbf{a})$.

The first-order perturbation of Eq. (10) can be given as

$$
\left(\mathbf{K}^{e}+\Delta \mathbf{K}\right)\left(\mathbf{U}_{j}^{e}+\Delta \mathbf{U}_{j}\right)=\left(\omega_{j}^{e}+\Delta \omega_{j}\right)^{2}\left(\mathbf{M}^{e}+\Delta \mathbf{M}\right)\left(\mathbf{U}_{j}^{e}+\Delta \mathbf{U}_{j}\right)
$$

where $\mathbf{U}_{j}^{e}$ and $\Delta \mathbf{U}_{j}$ are the mean value and deviation value of $\mathbf{U}_{j}(\mathbf{a}), \omega_{j}^{e}$ and $\Delta \omega_{j}$ are the mean value and deviation value of $\omega_{j}(\mathbf{a})$.

Due to the compact supports of FEM shape functions, it can be easily obtained that $\mathrm{K}$ and $\mathrm{M}$ are symmetric [44]. Pre-multiplying both sides of Eq. (13) by $\mathbf{U}_{j}^{e^{T}}$, using the normalization condition and ignoring the higher-order terms, $\Delta \omega_{j}$ can be given as

$$
\Delta \omega_{j}=\frac{1}{2 \omega_{j}^{e}} \mathbf{U}_{j}^{e^{T}}\left[\Delta \mathbf{K}-\omega_{j}^{e^{2}} \Delta \mathbf{M}\right] \mathbf{U}_{j}^{e}
$$

Therefore, the random natural angular frequency $\omega_{j}(\mathbf{a})$ can be expressed as

$$
\omega_{j}(\mathbf{a})=\omega_{j}^{e}+\Delta \omega_{j}=\omega_{j}^{e}+\sum_{i=1}^{m} \frac{1}{2 \omega_{j}^{e}} \mathbf{U}_{j}^{e^{T}}\left[\frac{\partial \mathbf{K}^{e}}{\partial a_{i}}-\omega_{j}^{e^{2}} \frac{\partial \mathbf{M}^{e}}{\partial a_{i}}\right] \mathbf{U}_{j}^{e}\left(a_{i}-a_{i}^{e}\right)
$$

Then, Eq. (15) can be rewritten as

$$
\omega_{j}(\mathbf{a})=B_{0}+\sum_{i=1}^{m} B_{i} a_{i}
$$

where

$$
\begin{aligned}
& B_{0}=\omega_{j}^{e}-\sum_{i=1}^{m} \frac{1}{2 \omega_{j}^{e}} \mathbf{U}_{j}^{e^{T}}\left[\frac{\partial \mathbf{K}^{e}}{\partial a_{i}}-\omega_{j}^{e^{2}} \frac{\partial \mathbf{M}^{e}}{\partial a_{i}}\right] \mathbf{U}_{j}^{e} a_{i}^{e} \\
& B_{i}=\frac{1}{2 \omega_{j}^{e}} \mathbf{U}_{j}^{e^{T}}\left[\frac{\partial \mathbf{K}^{e}}{\partial a_{i}}-\omega_{j}^{e^{2}} \frac{\partial \mathbf{M}^{e}}{\partial a_{i}}\right] \mathbf{U}_{j}^{e}
\end{aligned}
$$


By using the change-of-variable technique mentioned in Section 2, the probability density function of the random natural angular frequency $\omega_{j}(\mathbf{a})$ can be given as

$$
p_{\omega_{j}(\mathbf{a})}\left(\omega_{j}(\mathbf{a})\right)= \begin{cases}\frac{1}{\left|B_{1}\right|} p_{a_{1}}\left(\frac{\omega_{j}(\mathbf{a})-B_{0}}{B_{1}}\right) & (m=1) \\ \frac{1}{\left|B_{2}\right|} \int_{a_{1}=-\infty}^{\infty} p_{a_{1}}\left(a_{1}\right) p_{a_{2}}\left(\frac{\omega_{j}(\mathbf{a})-B_{0}-B_{1} a_{1}}{B_{2}}\right) \mathrm{d} a_{1}(m=2) \\ \int_{a_{m}=-\infty}^{\infty} p_{a_{m}}\left(a_{m}\right) p_{F_{m-2}}\left(\omega_{j}(\mathbf{a})-B_{0}-B_{m} a_{m}\right) \mathrm{d} a_{m} & (m>2)\end{cases}
$$

where $F_{m-2}=\sum_{i=1}^{m-1} B_{i} a_{i}$.

The natural frequency $f_{j}$ can be given as

$$
f_{j}=\frac{\omega_{j}}{2 \pi}
$$

Similarly, based on the change-of-variable technique, the probability density function of the random natural frequency $f_{j}(\mathbf{a})$ can be given as

$$
p_{f_{j}(\mathbf{a})}\left(f_{j}(\mathbf{a})\right)=2 \pi p_{\omega_{j}(\mathbf{a})}\left(2 \pi f_{j}(\mathbf{a})\right)
$$

\subsection{CVPS-FEM for mode shape of AMs with random parameters}

For convenience, Eq. (10) can be rewritten as

$$
\mathbf{K}(\mathbf{a}) \mathbf{U}_{j}(\mathbf{a})=\lambda_{j}(\mathbf{a}) \mathbf{M}(\mathbf{a}) \mathbf{U}_{j}(\mathbf{a})
$$

where $\lambda_{j}(\mathbf{a})=\omega_{j}^{2}(\mathbf{a})$ is the $j^{\text {th }}$ eigenvalue of AMs unit cell.

The first-order perturbation of Eq. (21) can be given as

$$
\left(\mathbf{K}^{e}+\Delta \mathbf{K}\right)\left(\mathbf{U}_{j}^{e}+\Delta \mathbf{U}_{j}\right)=\left(\lambda_{j}^{e}+\Delta \lambda_{j}\right)\left(\mathbf{M}^{e}+\Delta \mathbf{M}\right)\left(\mathbf{U}_{j}^{e}+\Delta \mathbf{U}_{j}\right)
$$

where $\lambda_{j}^{e}$ and $\Delta \lambda_{j}$ are the mean value and deviation value of $\lambda_{j}(\mathbf{a})$, respectively. 


$$
\mathbf{K}^{e} \Delta \mathbf{U}_{j}+\Delta \mathbf{K} \mathbf{U}_{j}^{e}=\lambda_{j}^{e} \mathbf{M} \Delta \mathbf{U}_{j}+\lambda_{j}^{e} \Delta \mathbf{M} \mathbf{U}_{j}^{e}+\Delta \lambda_{j} \mathbf{M}^{e} \mathbf{U}_{j}^{e}
$$

The deviation value of the $j^{\text {th }}$ mode shape $\Delta \mathbf{U}_{j}$ can be given as the superposition of these deterministic mode shapes [45]

$$
\Delta \mathbf{U}_{j}=\sum_{q=1}^{n} C_{q} \mathbf{U}_{q}^{e}
$$

where $C_{q}$ is the constant parameter with respect to $q^{\text {th }}$ mode shape $\mathbf{U}_{q}^{e}$.

Substituting Eq. (24) into Eq. (23) and pre-multiplying each side of Eq. (23) by $\mathbf{U}_{q}^{e^{T}}$, the following equation can be obtained based on the normalization condition.

$$
C_{q} \lambda_{q}^{e}+\mathbf{U}_{q}^{e^{T}} \Delta \mathbf{K} \mathbf{U}_{j}^{e}=C_{q} \lambda_{j}^{e}+\lambda_{j}^{e} \mathbf{U}_{q}^{e^{T}} \Delta \mathbf{M} \mathbf{U}_{j}^{e}
$$

When $q \neq j$, according to Eq. (25), $C_{q}$ can be calculated as

$$
C_{q}=\frac{\mathbf{U}_{q}^{e^{T}}\left(\Delta \mathbf{K}-\lambda_{j}^{e} \Delta \mathbf{M}\right) \mathbf{U}_{j}^{e}}{\lambda_{j}^{e}-\lambda_{q}^{e}}(q \neq j)
$$

When $q=j$, the first-order perturbation of Eq. (9) can be given as

$$
\left(\mathbf{U}_{j}^{e}+\Delta \mathbf{U}_{j}\right)^{T}\left(\mathbf{M}^{e}+\Delta \mathbf{M}\right)\left(\mathbf{U}_{j}^{e}+\Delta \mathbf{U}_{j}\right)=1
$$

Ignoring the higher-order terms, Eq. (27) can be rewritten as

$$
\mathbf{U}_{j}^{e^{T}} \mathbf{M}^{e} \Delta \mathbf{U}_{j}+\mathbf{U}_{j}^{e^{T}} \Delta \mathbf{M} \mathbf{U}_{j}^{e}+\Delta \mathbf{U}_{j}^{T} \mathbf{M}^{e} \mathbf{U}_{j}^{e}=0
$$

Pre-multiplying each side of Eq. (24) by $\mathbf{U}_{j}^{e^{T}} \mathbf{M}^{e}$, gives

$$
C_{q}=\mathbf{U}_{j}^{e^{T}} \mathbf{M}^{e} \Delta \mathbf{U}_{j}(q=j)
$$

Substituting Eq. (29) and its transpose into Eq. (28), $C_{q}$ can be given as

$$
C_{q}=-\frac{1}{2} \mathbf{U}_{j}^{e^{T}} \Delta \mathbf{M} \mathbf{U}_{j}^{e}(q=j)
$$

Substituting Eqs. (26) and (30) into Eq. (24), gives

$$
\Delta \mathbf{U}_{j}=\sum_{\substack{q=1 \\ q \neq j}}^{n} \frac{\mathbf{U}_{q}^{e^{T}}\left(\Delta \mathbf{K}-\lambda_{j}^{e} \Delta \mathbf{M}\right) \mathbf{U}_{j}^{e}}{\lambda_{j}^{e}-\lambda_{q}^{e}} \mathbf{U}_{q}^{e}-\frac{1}{2} \mathbf{U}_{j}^{e^{T}} \Delta \mathbf{M} \mathbf{U}_{j}^{e} \mathbf{U}_{j}^{e}
$$


Therefore, the random mode shape $\mathbf{U}_{j}(\mathbf{a})$ can be expressed as

$$
\begin{aligned}
\mathbf{U}_{j}(\mathbf{a}) & =\mathbf{U}_{j}^{e}+\Delta \mathbf{U}_{j} \\
& =\mathbf{U}_{j}^{e}+\sum_{i=1}^{m}\left[\left(\sum_{\substack{q=1 \\
q \neq j}}^{n} \frac{\mathbf{U}_{q}^{e^{T}}\left(\frac{\partial \mathbf{K}^{e}}{\partial a_{i}}-\lambda_{j}^{e} \frac{\partial \mathbf{M}^{e}}{\partial a_{i}}\right) \mathbf{U}_{j}^{e}}{\lambda_{j}^{e}-\lambda_{q}^{e}} \mathbf{U}_{q}^{e}-\frac{1}{2} \mathbf{U}_{j}^{e^{T}} \frac{\partial \mathbf{M}^{e}}{\partial a_{i}} \mathbf{U}_{j}^{e} \mathbf{U}_{j}^{e}\right)\left(a_{i}-a_{i}^{e}\right)\right]
\end{aligned}
$$

Then, Eq. (32) can be rewritten as

$$
\mathbf{U}_{j}(\mathbf{a})=\mathbf{D}_{0}+\sum_{i=1}^{m} \mathbf{D}_{i} a_{i}
$$

where

$$
\begin{aligned}
& \mathbf{D}_{0}=\mathbf{U}_{j}^{e}-\sum_{i=1}^{m}\left[\left(\sum_{\substack{q=1 \\
q \neq j}}^{n} \frac{\mathbf{U}_{q}^{e^{T}}\left(\frac{\partial \mathbf{K}^{e}}{\partial a_{i}}-\lambda_{j}^{e} \frac{\partial \mathbf{M}^{e}}{\partial a_{i}}\right) \mathbf{U}_{j}^{e}}{\lambda_{j}^{e}-\lambda_{q}^{e}} \mathbf{U}_{q}^{e}-\frac{1}{2} \mathbf{U}_{j}^{e^{T}} \frac{\partial \mathbf{M}^{e}}{\partial a_{i}} \mathbf{U}_{j}^{e} \mathbf{U}_{j}^{e}\right] a_{i}^{e}\right] \\
& \mathbf{D}_{i}=\sum_{\substack{q=1 \\
q \neq j}}^{n} \frac{\mathbf{U}_{q}^{e^{T}}\left(\frac{\partial \mathbf{K}^{e}}{\partial a_{i}}-\lambda_{j}^{e} \frac{\partial \mathbf{M}^{e}}{\partial a_{i}}\right) \mathbf{U}_{j}^{e}}{\lambda_{j}^{e}-\lambda_{q}^{e}} \mathbf{U}_{q}^{e}-\frac{1}{2} \mathbf{U}_{j}^{e^{T}} \frac{\partial \mathbf{M}^{e}}{\partial a_{i}} \mathbf{U}_{j}^{e} \mathbf{U}_{j}^{e}
\end{aligned}
$$

The random mode shape $\mathbf{U}_{j}(\mathbf{a})$ can be given in another more apparent vector forms as

$$
\mathbf{U}_{j}(\mathbf{a})=\left\{\begin{array}{c}
U_{j, 1}(\mathbf{a}) \\
\mathbf{M} \\
U_{j, k}(\mathbf{a}) \\
\mathbf{M} \\
U_{j, n}(\mathbf{a})
\end{array}\right\} ; \mathbf{D}_{0}=\left\{\begin{array}{r}
D_{0,1} \\
\mathbf{M} \\
D_{0, k} \\
\mathbf{M} \\
D_{0, n}
\end{array}\right\} ; \mathbf{D}_{i}=\left\{\begin{array}{r}
D_{i, 1} \\
\mathbf{M} \\
D_{i, k} \\
\mathbf{M} \\
D_{i, n}
\end{array}\right\} \quad \mathbf{U}_{j}(\mathbf{a})=\mathbf{D}_{0}+\sum_{i=1}^{m} \mathbf{D}_{i} a_{i}
$$

The random displacement $U_{j k}(\mathbf{a})$ of $k^{\text {th }}$ degree in the $j^{\text {th }}$ mode shape can be given as

$$
U_{j k}(\mathbf{a})=D_{0, k}+\sum_{i=1}^{m} D_{i, k} a_{i}
$$

By using the change-of-variable technique mentioned in Section 2, the probability density function of the random displacement $U_{j k}(\mathbf{a})$ can be given as 


$$
p_{U_{j k}(\mathbf{a})}\left(U_{j k}(\mathbf{a})\right)= \begin{cases}\frac{1}{\left|D_{1}\right|} p_{a_{1}}\left(\frac{U_{j k}(\mathbf{a})-D_{0}}{D_{1}}\right) & (m=1) \\ \frac{1}{\left|D_{2}\right|} \int_{a_{1}=-\infty}^{\infty} p_{a_{1}}\left(a_{1}\right) p_{a_{2}}\left(\frac{U_{j k}(\mathbf{a})-D_{0}-D_{1} a_{1}}{D_{2}}\right) \mathrm{d} a_{1}(m=2) & (m) p^{\infty} p_{a_{m}}\left(a_{m}\right) p_{G_{m-2}}\left(U_{j k}(\mathbf{a})-D_{0}-D_{m} a_{m}\right) \mathrm{d} a_{m} \quad(m>2)\end{cases}
$$

where $G_{m-2}=\sum_{i=1}^{m-1} D_{i} a_{i}$.

\subsection{CVPS-FEM for frequency response function of AMs with random parameters}

The structural dynamic equilibrium equation of AMs model can be given as

$$
\left(\mathbf{K}-\omega^{2} \mathbf{M}\right) \mathbf{R}=\mathbf{F}
$$

where $\mathbf{F}$ and $\omega$ are the external unit load and the corresponding angular frequency, respectively. $\mathbf{R}$ donates the displacement response of the AMs.

To simplify the calculation process, Eq. (38) can be rewritten as

$$
\mathbf{Z R}=\mathbf{F}
$$

where $\mathbf{Z}$ is the structural dynamic stiffness matrix of the AMs and can be given as

$$
\mathbf{Z}=\mathbf{K}-\omega^{2} \mathbf{M}
$$

When the random parameters are introduced, the structural dynamic equilibrium equation of AMs with the random parameters can be given as

$$
\mathbf{Z}(\mathbf{a}) \mathbf{R}(\mathbf{a})=\mathbf{F}(\mathbf{a})
$$

Similarly, based on the first-order Taylor expansion, $\mathbf{Z}(\mathbf{a})$ and $\mathbf{F}(\mathbf{a})$ can be developed as

$$
\mathbf{Z}(\mathbf{a})=\mathbf{Z}\left(\mathbf{a}^{e}\right)+\sum_{i=1}^{m} \frac{\partial \mathbf{Z}\left(\mathbf{a}^{e}\right)}{\partial a_{i}}\left(a_{i}-a_{i}^{e}\right)=\mathbf{Z}^{e}+\Delta \mathbf{Z}
$$




$$
\mathbf{F}(\mathbf{a})=\mathbf{F}\left(\mathbf{a}^{e}\right)+\sum_{i=1}^{m} \frac{\partial \mathbf{F}\left(\mathbf{a}^{e}\right)}{\partial a_{i}}\left(a_{i}-a_{i}^{e}\right)=\mathbf{F}^{e}+\Delta \mathbf{F}
$$

where $\mathbf{Z}^{e}$ and $\Delta \mathbf{Z}$ are the mean value and deviation value of $\mathbf{Z}(\mathbf{a}), \mathbf{F}^{e}$ and $\Delta \mathbf{F}$ are the mean value and deviation value of $\mathbf{F}(\mathbf{a})$.

The first-order perturbation of Eq. (41) can be given as

$$
\left(\mathbf{Z}^{e}+\Delta \mathbf{Z}\right)\left(\mathbf{R}^{e}+\Delta \mathbf{R}\right)=\left(\mathbf{F}^{e}+\Delta \mathbf{F}\right)
$$

Neglecting the high-order terms, $\Delta \mathbf{R}$ can be computed as [43]

$$
\Delta \mathbf{R}=\left(\mathbf{Z}^{e}\right)^{-1}\left(\Delta \mathbf{F}-\Delta \mathbf{Z} \mathbf{R}^{e}\right)
$$

Therefore, $\mathbf{R}(\mathbf{a})$ can be expressed as

$$
\mathbf{R}(\mathbf{a})=\mathbf{R}^{e}+\Delta \mathbf{R}=\mathbf{R}^{e}+\sum_{i=1}^{m}\left(\mathbf{Z}^{e}\right)^{-1}\left(\frac{\partial \mathbf{F}^{e}}{\partial a_{i}}-\frac{\partial \mathbf{Z}^{e}}{\partial a_{i}} \mathbf{R}^{e}\right)\left(a_{i}-a_{i}^{e}\right)
$$

Eq. (46) can be rewritten as

$$
\mathbf{R}(\mathbf{a})=\mathbf{H}_{0}+\sum_{i=1}^{m} \mathbf{H}_{i} a_{i}
$$

where

$$
\begin{aligned}
& \mathbf{H}=\mathbf{R}^{e}-\sum_{i=1}^{m}\left(\mathbf{Z}^{e}\right)^{-1}\left(\frac{\partial \mathbf{F}^{e}}{\partial a_{i}}-\frac{\partial \mathbf{Z}^{e}}{\partial a_{i}} \mathbf{R}^{e}\right) a_{i}^{e} \\
& \mathbf{H}_{i}=\left(\mathbf{Z}^{e}\right)^{-1}\left(\frac{\partial \mathbf{F}^{e}}{\partial a_{i}}-\frac{\partial \mathbf{Z}^{e}}{\partial a_{i}} \mathbf{R}^{e}\right)
\end{aligned}
$$

The random displacement response $\mathbf{R}(\mathbf{a})$ can be given in another more apparent vector forms as

$$
\mathbf{R}(\mathbf{a})=\left\{\begin{array}{c}
R_{1}(\mathbf{a}) \\
\mathrm{M} \\
R_{k}(\mathbf{a}) \\
\mathrm{M} \\
R_{n}(\mathbf{a})
\end{array}\right\} ; \mathbf{H}_{0}=\left\{\begin{array}{r}
H_{0,1} \\
\mathrm{M} \\
H_{0, k} \\
\mathrm{M} \\
H_{0, n}
\end{array}\right\} ; \mathbf{H}_{i}=\left\{\begin{array}{r}
H_{i, 1} \\
\mathrm{M} \\
H_{i, k} \\
\mathrm{M} \\
H_{i, n}
\end{array}\right\} \quad \mathbf{R}(\mathbf{a})=\mathbf{H}_{0}+\sum_{i=1}^{m} \mathbf{H}_{i} a_{i}
$$

The random displacement response $R_{k}(\mathbf{a})$ of $k^{\text {th }}$ degree can be expressed as 


$$
R_{k}(\mathbf{a})=\mathrm{H}_{0, k}+\sum_{i=1}^{m} \mathrm{H}_{i, k} a_{i}
$$

Similarly, by using the change-of-variable technique mentioned in Section 2, the probability density function of the random displacement response $R_{k}(\mathbf{a})$ can be given as

$$
p_{\mathrm{R}_{k}(\mathbf{a})}\left(R_{k}(\mathbf{a})\right)= \begin{cases}\frac{1}{\left|H_{1}\right|} p_{a_{1}}\left(\frac{R_{k}(\mathbf{a})-H_{0}}{H_{1}}\right) & (m=1) \\ \frac{1}{\left|H_{2}\right|} \int_{a_{1}=-\infty}^{\infty} p_{a_{1}}\left(a_{1}\right) p_{a_{2}}\left(\frac{R_{k}(\mathbf{a})-H_{0}-H_{1} a_{1}}{H_{2}}\right) \mathrm{d} a_{1}(m=2) \\ \int_{a_{m}=-\infty}^{\infty} p_{a_{m}}\left(a_{m}\right) p_{S_{m-2}}\left(R_{k}(\mathbf{a})-H_{0}-H_{m} a_{m}\right) \mathrm{d} a_{m} & (m>2)\end{cases}
$$

where $S_{m-2}=\sum_{i=1}^{m-1} H_{i} a_{i}$.

\section{Numerical examples}

In this section, three numerical examples are employed to illustrate the effectiveness of the formulated CVPS-FEM model. The probability distributions of band structures, mode shapes, and frequency responses for three different types stochastic AMs are predicted in these numerical examples. Furthermore, the accuracy of the results obtained by CVPS-FEM is verified by MCM. The material properties for the random structure in this paper are given as follows:

\section{Table 1}

Random material properties for AMs in numerical examples. (Young's modulus $E$, Poisson's ratio $v$, density $\rho$ )

\begin{tabular}{cllll}
\hline Material & Random variable & Distribution type & Parameters of random variable \\
\hline \multirow{2}{*}{ Lead } & $E(\mathrm{~Pa})$ & Gaussian distribution & $\mu_{l, E}=40.8 \times e 9$ & $\sigma_{l, E}=8 \times e 8$ \\
& $v$ & Log-normal distribution & $\mu_{l, v}=-0.9948$ & $\sigma_{l, v}=0.0329$
\end{tabular}




\begin{tabular}{lllll} 
& $\rho\left(\mathrm{kg} / \mathrm{m}^{3}\right)$ & Gaussian distribution & $\mu_{l, \rho}=11600$ & $\sigma_{l, \rho}=150$ \\
\hline \multirow{2}{*}{ Epoxy } & $E(\mathrm{~Pa})$ & Gaussian distribution & $\mu_{e, E}=3.6 \times e 9$ & $\sigma_{e, E}=8 \times e 7$ \\
& $\rho$ & Log-normal distribution & $\mu_{e, v}=-0.9947$ & $\sigma_{e, v}=0.0285$ \\
& $\rho\left(\mathrm{kg} / \mathrm{m}^{3}\right)$ & Gaussian distribution & $\mu_{e, \rho}=1180$ & $\sigma_{e, \rho}=20$ \\
\hline \multirow{2}{*}{ Rubber } & $E(\mathrm{~Pa})$ & Gaussian distribution & $\mu_{r, E}=11.75 \times e 4$ & $\sigma_{r, E}=3 \times e 3$ \\
& $v$ & Log-normal distribution & $\mu_{r, v}=-0.7572$ & $\sigma_{r, v}=0.0046$ \\
& $\rho\left(\mathrm{kg} / \mathrm{m}^{3}\right)$ & Gaussian distribution & $\mu_{r, \rho}=1300$ & $\sigma_{r, \rho}=22$ \\
\hline
\end{tabular}

\subsection{Binary AMs with hexagon lattice}

Consider uncertain prediction of the physical response for a Bragg-type AMs with hexagon lattice as shown in Fig. 2(a), and the microstructure is made of epoxy with periodical circular-shaped lead inclusions. In this numerical example, the structure parameters are given as follows: lattice constant $a=0.086603 \mathrm{~m}$, radius of lead columns $r=0.03 \mathrm{~m}$, length side of the hexagon unit cell $l=0.05 \mathrm{~m}$, and the material properties of epoxy and lead are defined as random parameters as presented in Table 1. To obtain the frequency response function of AMs, a simplified AMs model composed of several unit cells is proposed as shown in Fig. 2(b). In this frequency response model, it can be seen that the unit forced displacement is applied to the lower side boundary in $y$ direction and the response is obtained at the center of the upper side boundary. 


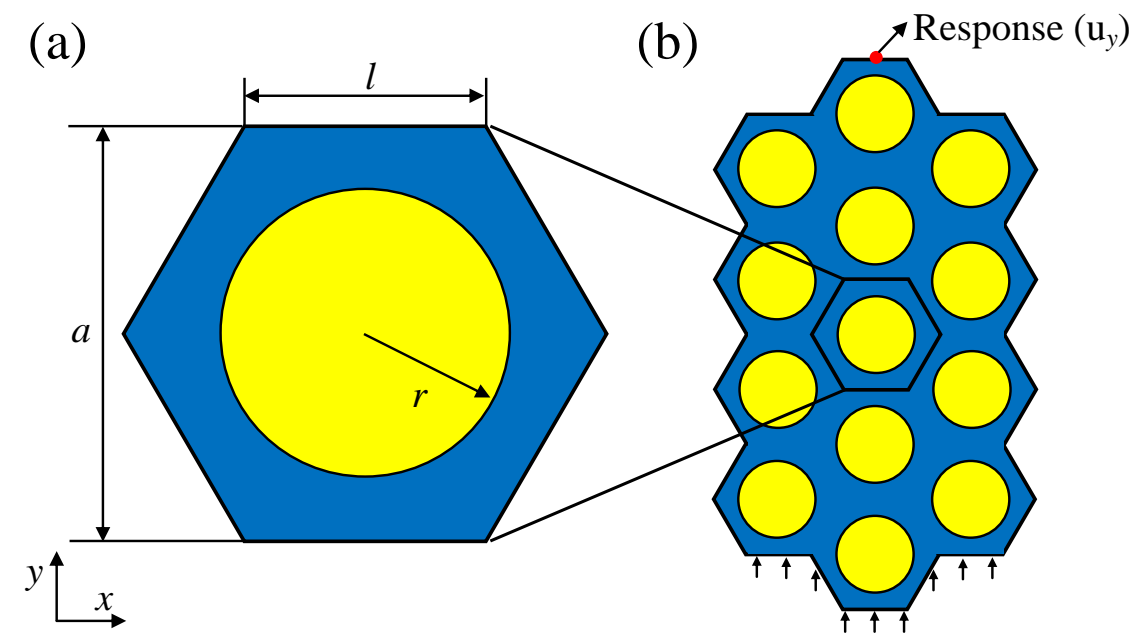

Fig. 2. Sketch of a cross-section of AMs with a hexagon lattice of inclusions with a circular cross-section. (a)

The unit cell of AMs and (b) the frequency response calculation model of AMs.

In Fig. 3, the band structure and frequency response function of AMs with mean properties are firstly calculated. As expected, the amplitude of frequency response is sharply dropped at the frequency range where the band gap exists.
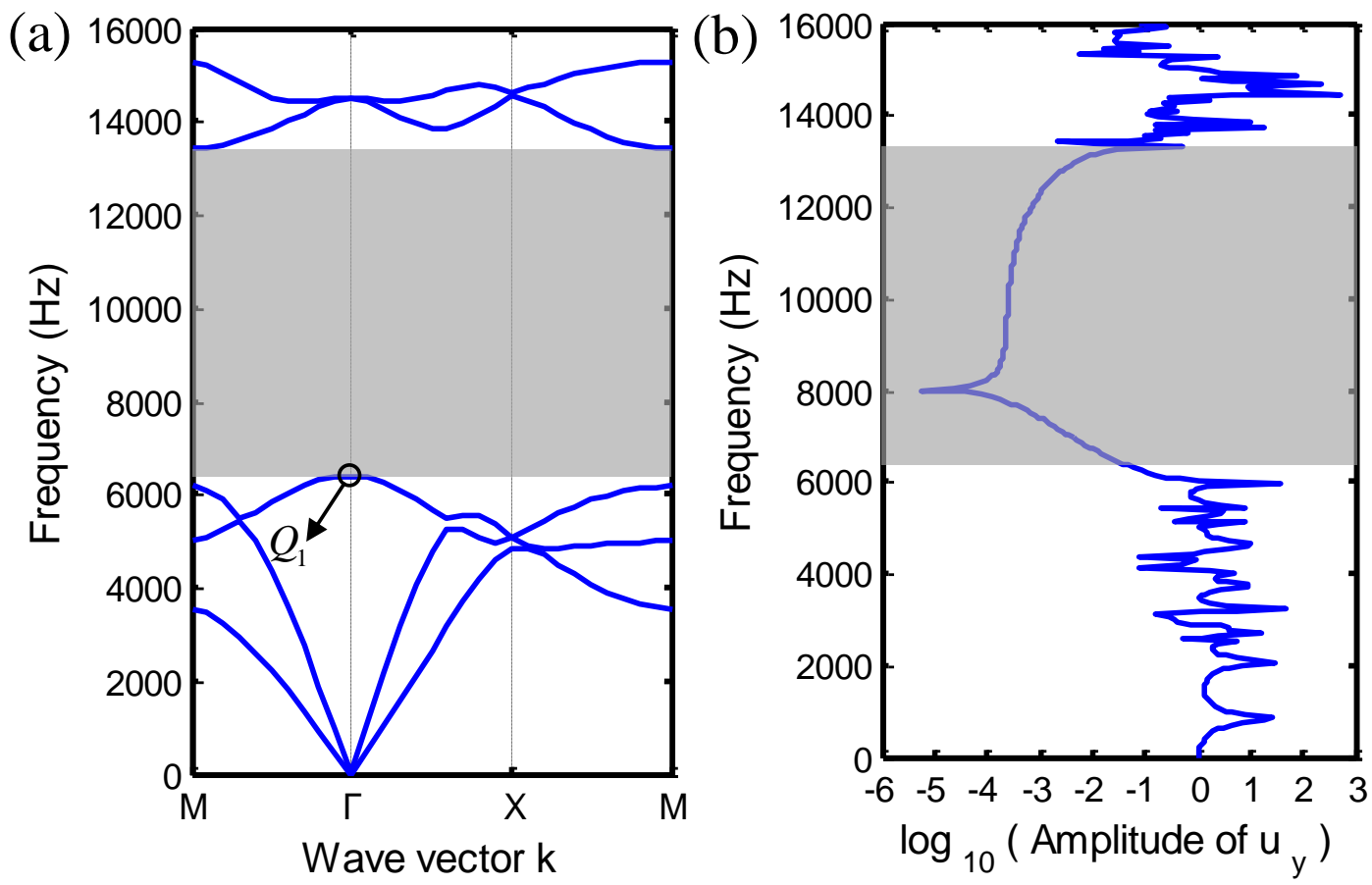

Fig. 3. The band structure and frequency response function of AMs with mean properties. (a) The band structure and (b) the frequency response function.

In this example, the Young's modulus $E$, Poisson's ratio $v$ and density $\rho$ of epoxy 
and lead are assumed to be random variables, and the parameters of these random variables are given in Table 1. In the following analysis, the uncertainty model based on CVPS-FEM is used to predict the probability density functions of band structure, mode shape and frequency response of the Bragg-type AMs with random parameters.

In the uncertain analysis of the band structure of AMs, the uncertain band gap is most concerned. The probability density function of the $3^{\text {rd }}$ natural frequency along $\Gamma$ direction (point $Q_{1}$ (lower boundary of the band gap) in Fig. 3(a)) of the stochastic AMs is calculated by CVPS-FEM, and it is shown in Fig. 4. To validate the accuracy of CVPS-FEM for the prediction of random band structure, the results obtained by MCM with 100000 samples are also given in Fig. 4. It can be seen from Fig. 4 that the probability density function calculated by CVPS-FEM matches the referenced results of MCM perfectly. Thus, it can be concluded that the proposed CVPS-FEM is very accurate to predict the physical response of AMs with random variables. Furthermore, it can be seen from Fig. 4 that the frequency value of point $Q_{1}$ of band structure has a large variation when the random parameters are introduced. Therefore, it is necessary to develop an accurate and fast model to predict the uncertain response of AMs. Similarly, the probability density function can also be obtained by CVPS-FEM for other random natural frequencies in the given directions.

In order to study the accuracy of CVPS-FEM for the random parameters with bigger variances, the Young's modulus and density with two times and three times standard deviation are applied, and the results of the probability density function of the $3^{\text {rd }}$ natural frequency along $\Gamma$ direction are given in Fig 5 . It can be observed clearly from Fig. 5 that the difference between CVPS-FEM and MCM results becomes larger when the bigger 
variance of the random parameters exist, which indicates that the formulated CVPS-FEM model of AMs is more suitable for small variance of random problems. The large variance of random parameters in the AMs can be considered as our future work.

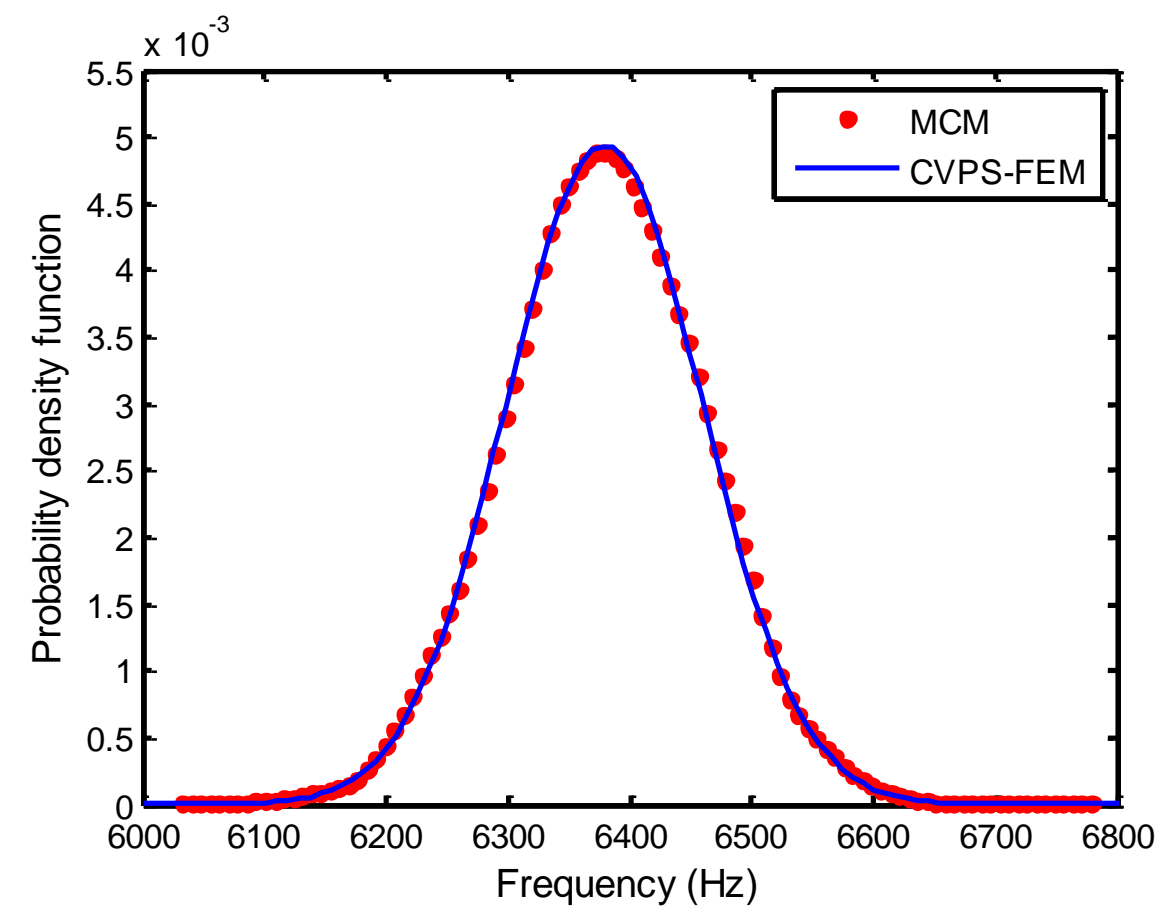

Fig. 4. The probability density function of the $3^{\text {rd }}$ natural frequency along $\Gamma$ direction $\left(Q_{1}\right)$ of AMs with random parameters.

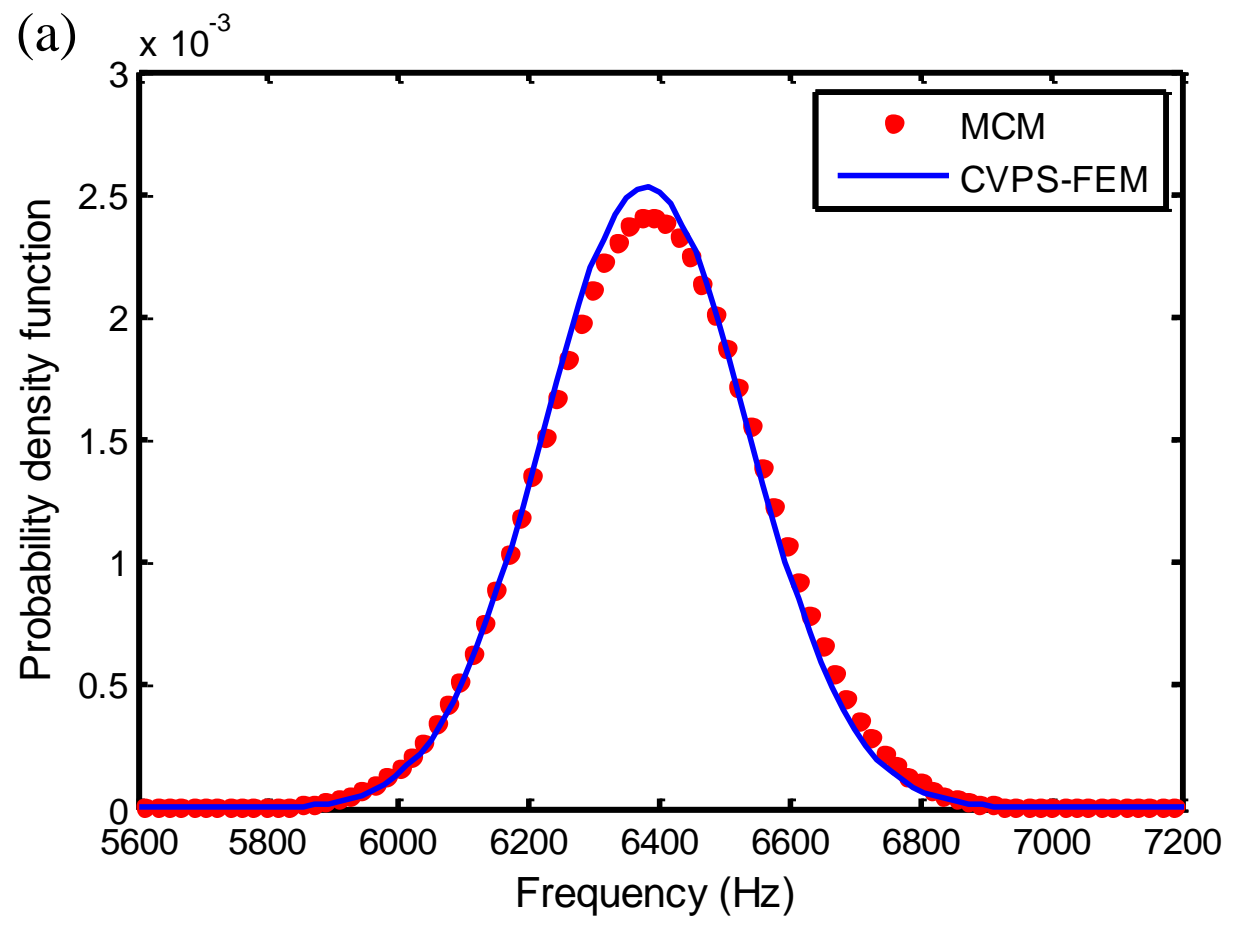




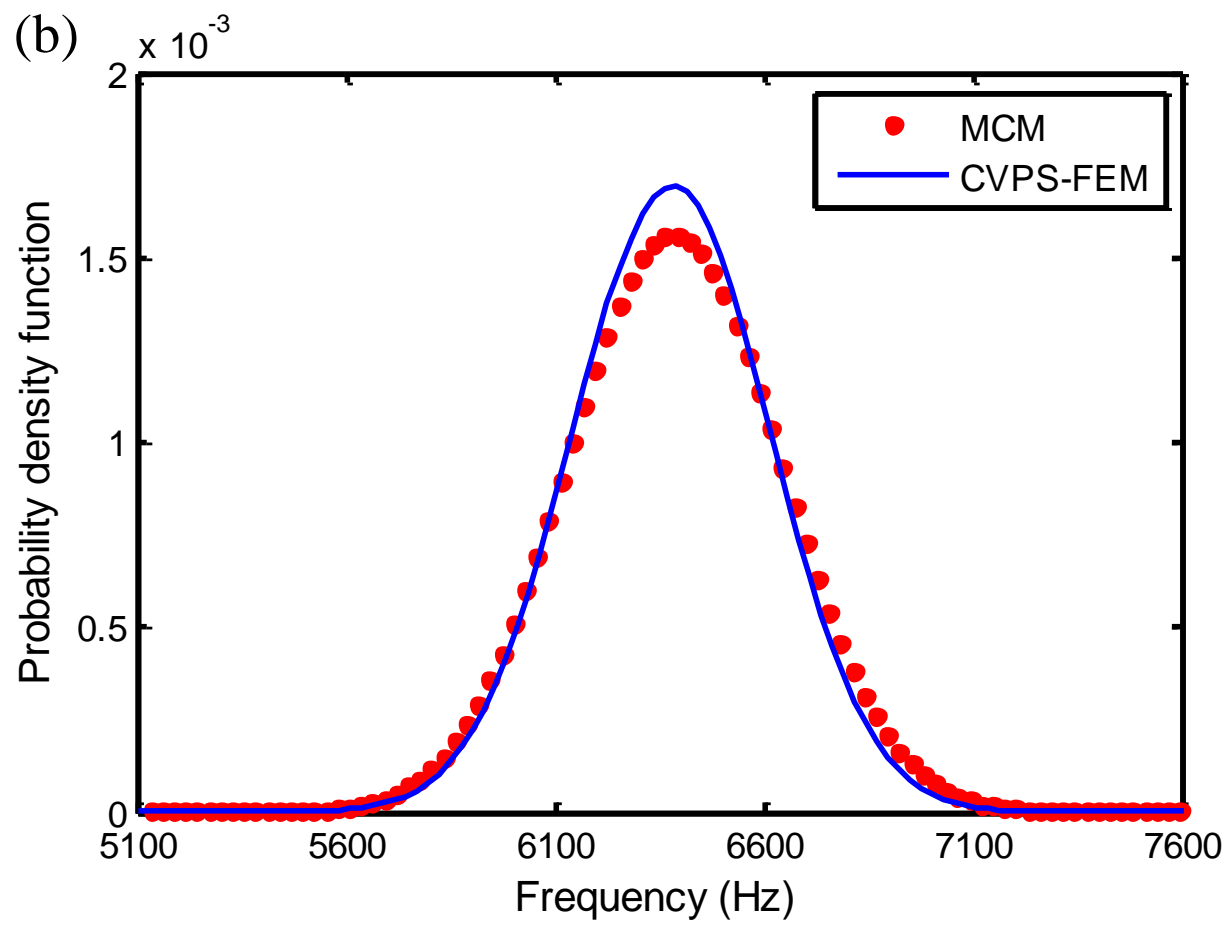

Fig. 5. The probability density function of the $3 \mathrm{rd}$ natural frequency along $\Gamma$ direction $\left(Q_{1}\right)$ of $\mathrm{AMs}$ with random parameters. (a) $\sigma_{l, E_{2}}=2 \sigma_{l, E}, \sigma_{l, \rho_{2}}=2 \sigma_{l, \rho}, \sigma_{e, E_{2}}=2 \sigma_{e, E}, \sigma_{e, \rho_{2}}=2 \sigma_{e, \rho}$; and (b) $\sigma_{l, E_{3}}=3 \sigma_{l, E}$, $\sigma_{l, \rho_{3}}=3 \sigma_{l, \rho}, \sigma_{e, E_{3}}=3 \sigma_{e, E}, \sigma_{e, \rho_{3}}=3 \sigma_{e, \rho}$

Next, the $3^{\text {rd }}$ mode shape along $\Gamma$ direction of the AMs unit cell with mean properties is analyzed as plotted in Fig. 6. Considering the uncertainty of the material properties, the probability density functions of the random normalized $x$-displacement and $y$-displacement of node $N_{1}$ in the 3rd mode shape are calculated by CVPS-FEM and MCM as shown in Fig. 7. It is clearly seen that the probability density varies with the normalized nodal displacements in $x$ and $y$ direction when the random parameters are introduced. It can also be observed that the uncertain results from CVPS-FEM are very reliable compared with the results obtained from MCM. Using the same approach, the probability density functions of the normalized nodal displacements in mode shape can also be predicted by CVPS-FEM for other specific locations of AMs with random parameters. 


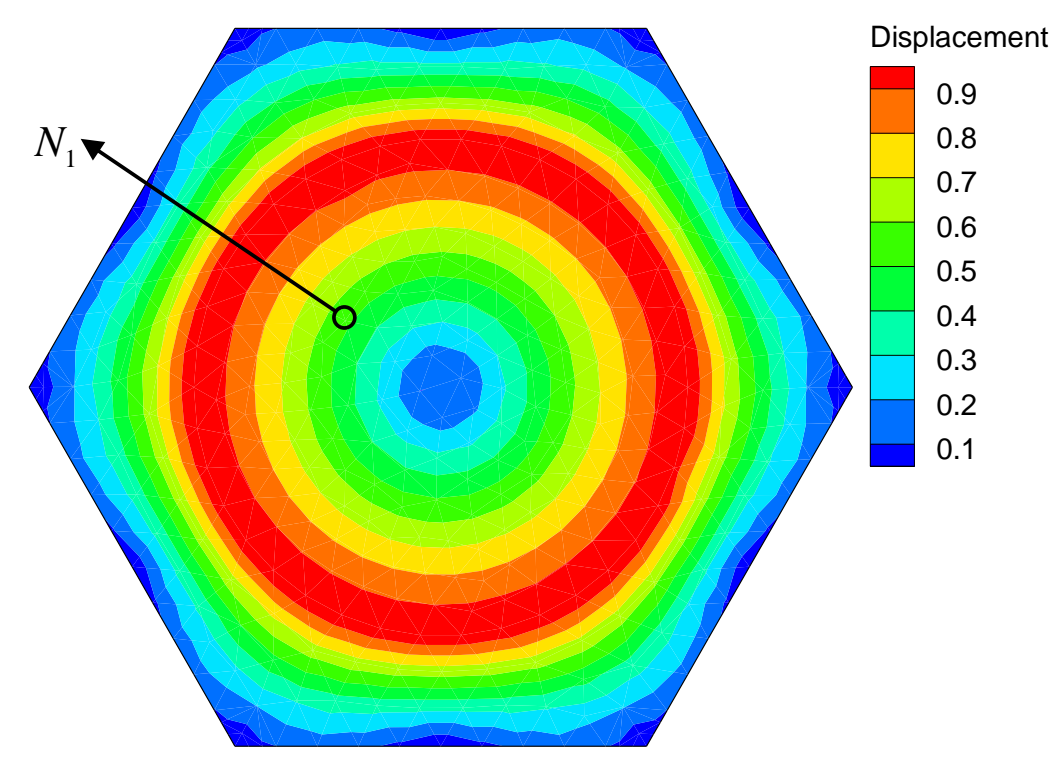

Fig. 6. The $3^{\text {rd }}$ mode shape along $\Gamma$ direction of AMs unit cell with mean properties.

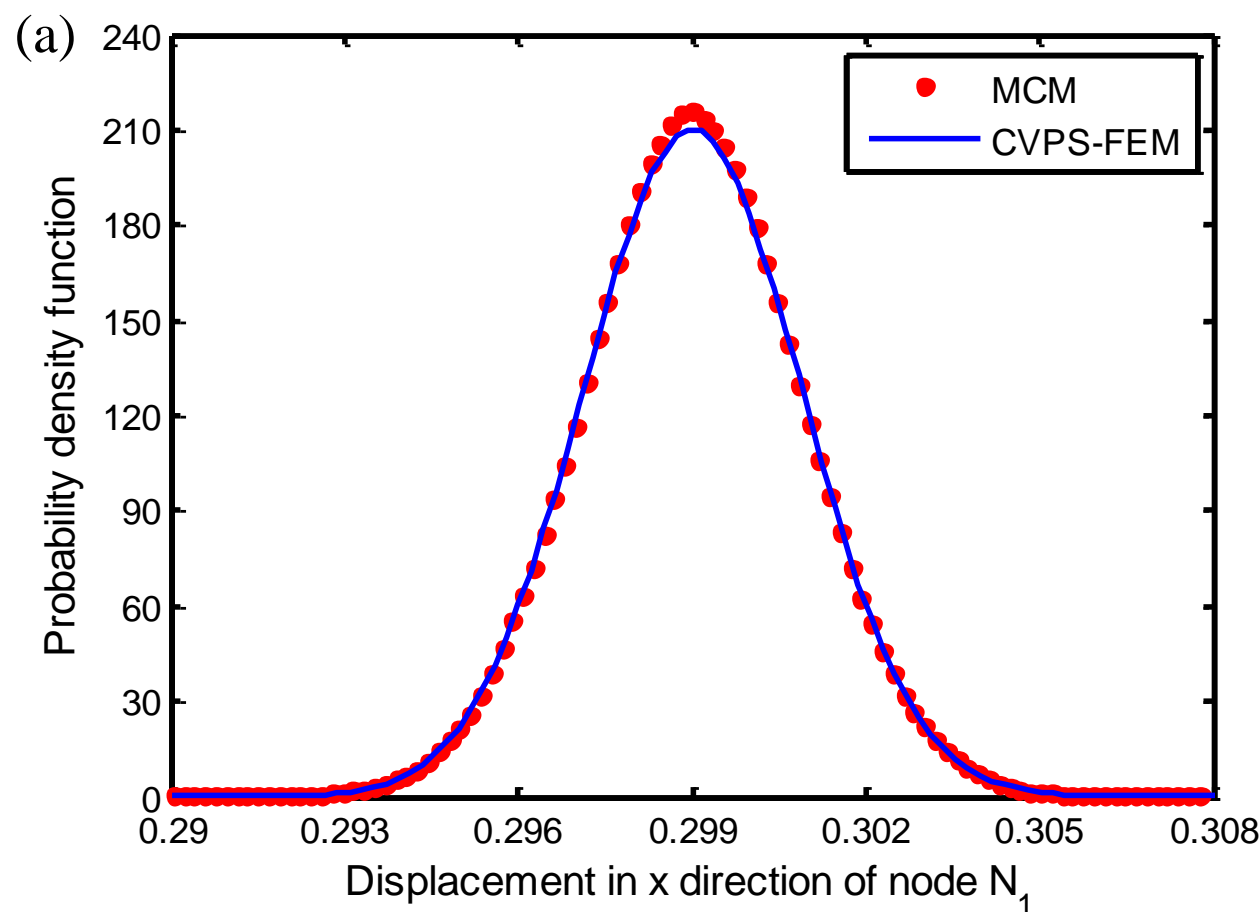




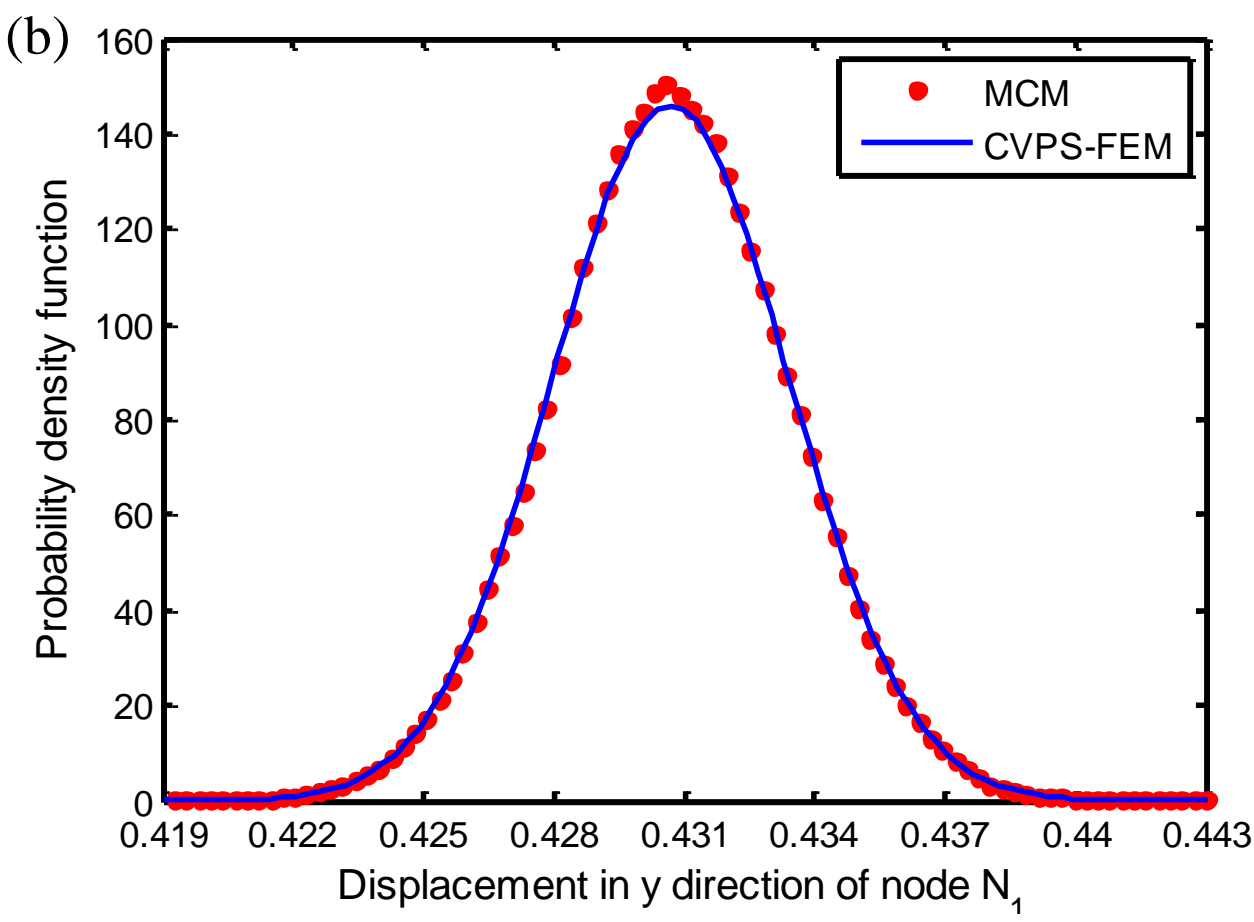

Fig. 7. The probability density function of the normalized nodal displacements in x and y direction of node $N_{1}$ of the $3^{\text {rd }}$ mode shape along $\Gamma$ direction of AMs unit cell with random parameters. (a) The normalized nodal displacement in $x$ direction and (b) the normalized nodal displacement in $y$ direction.

Figure 8 illustrates the probability density function of the frequency response using CVPS-FEM and MCM models at $200 \mathrm{~Hz}$. The numerical solutions of the probability density function obtained from CVPS-FEM and MCM models are almost identical. It is obviously noticed that the $95 \%$ confidence interval for the response amplitudes is about 1.073 to 1.083 . 


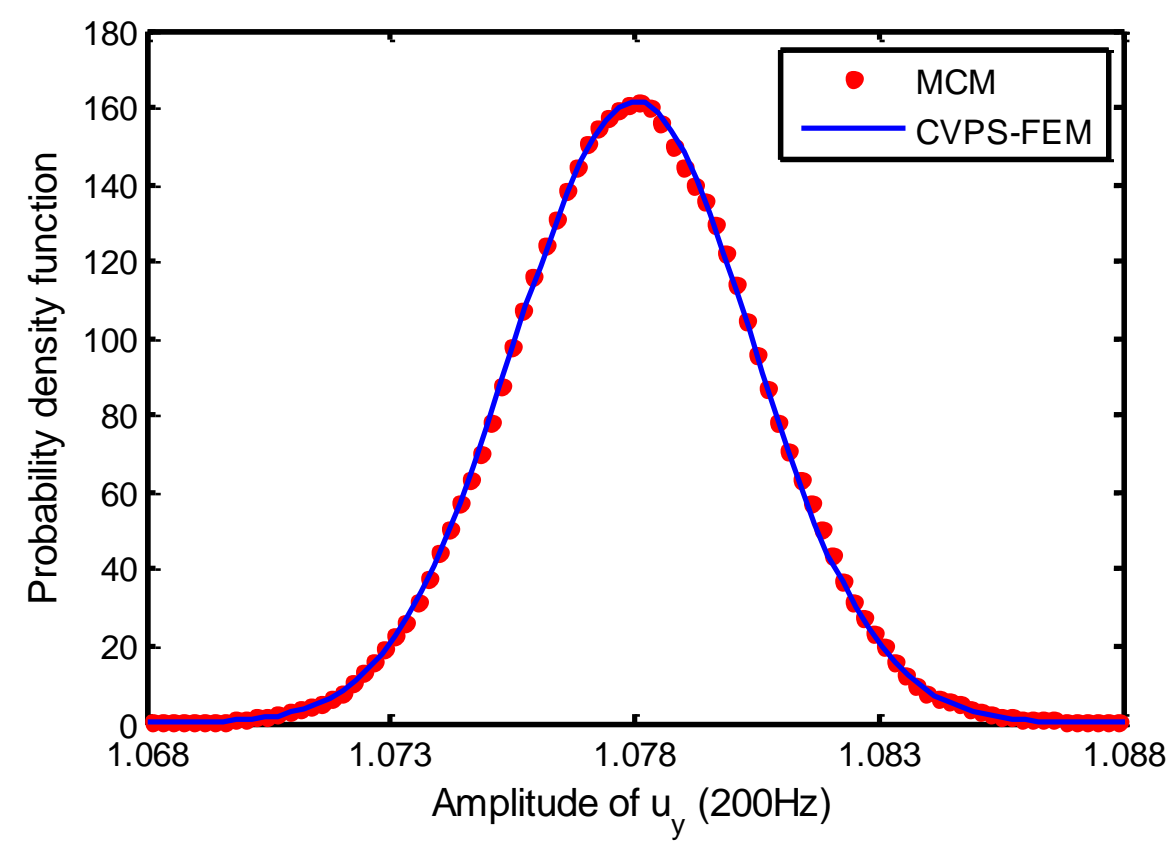

Fig. 8. The probability density function of the frequency response at $200 \mathrm{~Hz}$ of AMs with random parameters.

\subsection{Ternary LRAMs with square lattice}

A ternary LRAMs with lattice constant $a=0.02 \mathrm{~m}$ shown in Fig. 9(a) is investigated in this example, and the structure is made up of epoxy with lead cylinders of radius $R_{\text {in }}=0.006 \mathrm{~m}$ coated with rubber, the external radius of rubber cylinders is $R_{\mathrm{ex}}=0.008 \mathrm{~m}$. The material properties for lead, rubber and epoxy are defined as random parameters, which are given in Table 1. As shown in Fig. 9(b), $5 \times 5$ unit cells of LRAMs are constructed to calculate the frequency response function. Similarly, the unit forced displacement is applied to the lower side boundary of the model in $y$ direction and the frequency response is obtained at the center of its upper side boundary. 


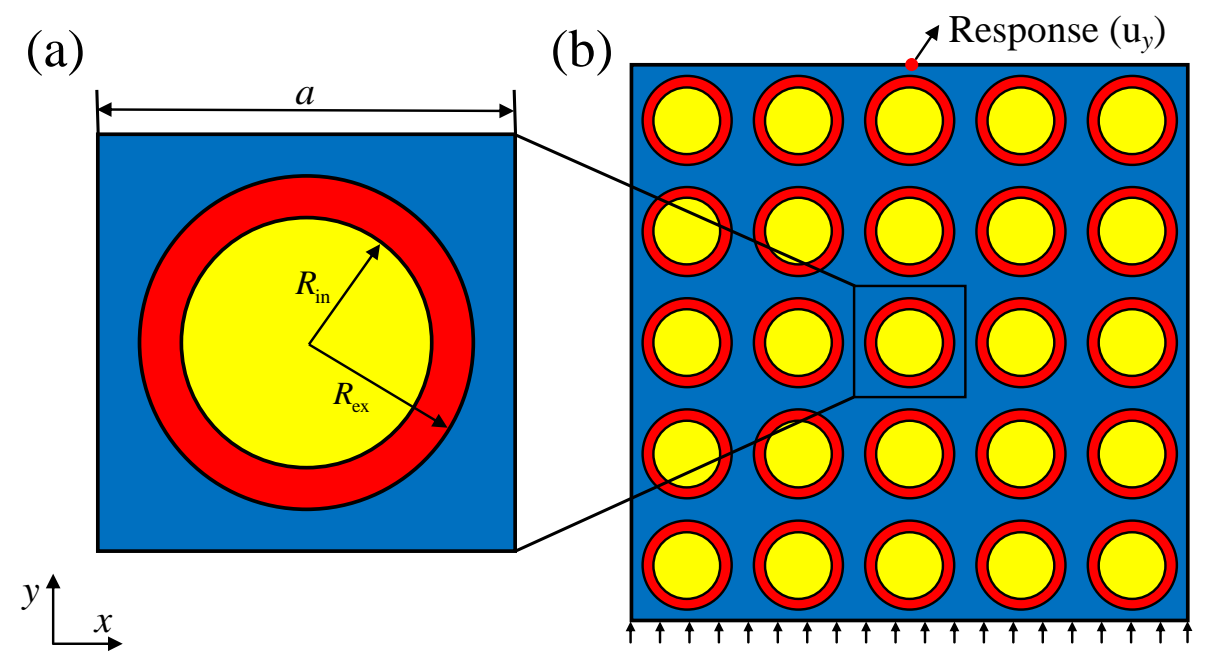

Fig. 9. Sketch of a cross-section of LRAMs with a square lattice of inclusions with a circular cross-section. (a)

The unit cell of LRAMs and (b) the frequency response calculation model of LRAMs.

First, the band structure and frequency function of LRAMs with mean properties are plotted in Fig. 10. As expected, it can be observed clearly from Fig. 10 that the amplitude of frequency response drops sharply when the band gaps exist, which indicates that LRAMs can reduce the vibration effectively.
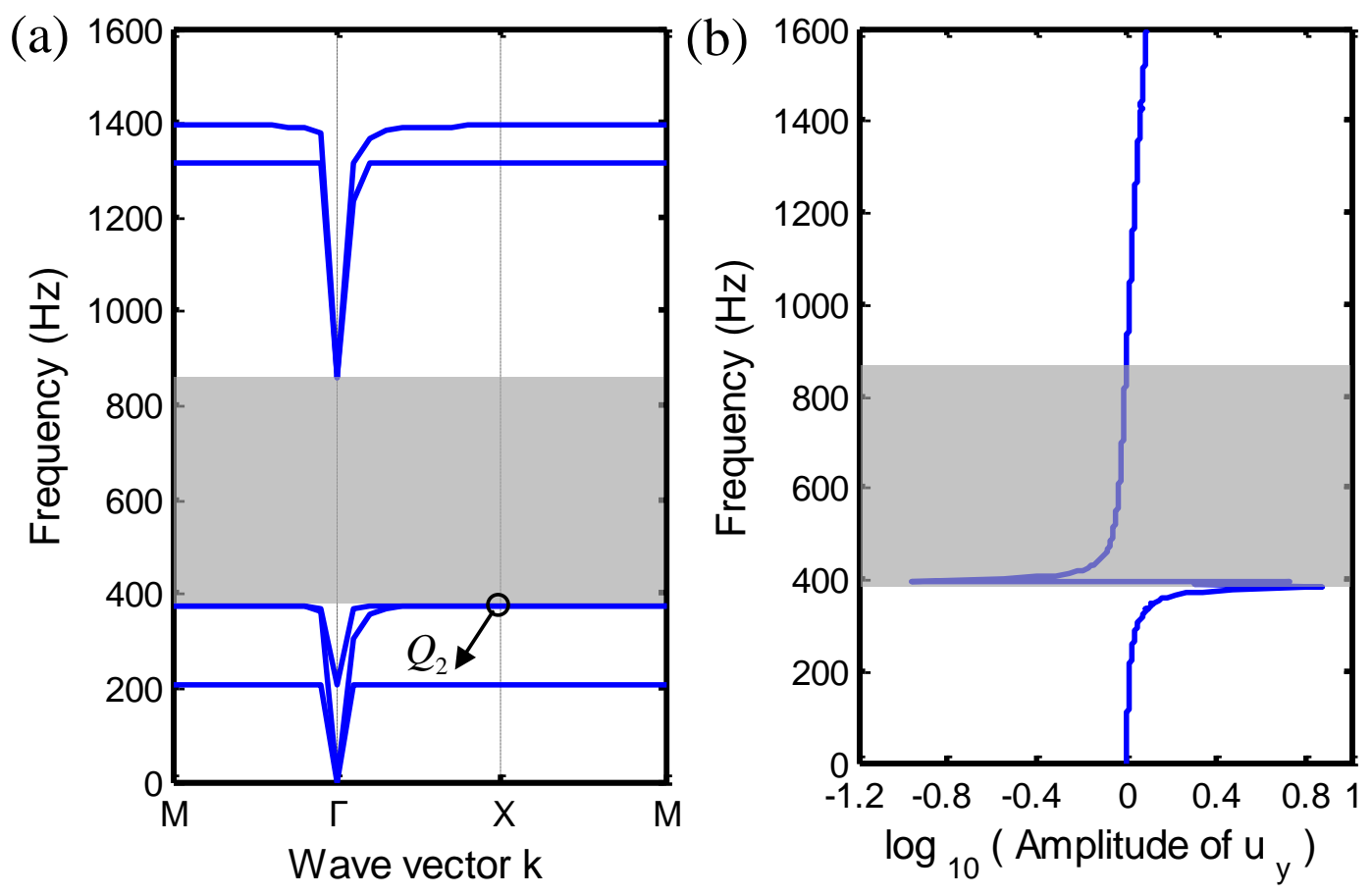

Fig. 10. The band structure and frequency response function of LRAMs with mean properties. (a) The band 
structure and (b) the frequency response function.

Then, consider the random uncertainty of material parameters of epoxy, rubber and lead, the uncertainty model based on CVPS-FEM is used to predict the probability density functions of the uncertain physical responses of LRAMs, and these random material parameters are listed in Table 1.

Figure 11 plots the probability density function of the random $3^{\text {rd }}$ natural frequency along X direction (point $Q_{2}$ (lower boundary of the band gap) in Fig. 10(a)) of LRAMs with random material properties calculated by CVPS-FEM and MCM. And the probability density function of random natural frequency computed by CVPS-FEM is in perfect agreement with the result obtained by the MCM, which clearly validates that CVPS-FEM is an effective method to predict the random band structure of LRAMs with random parameters.

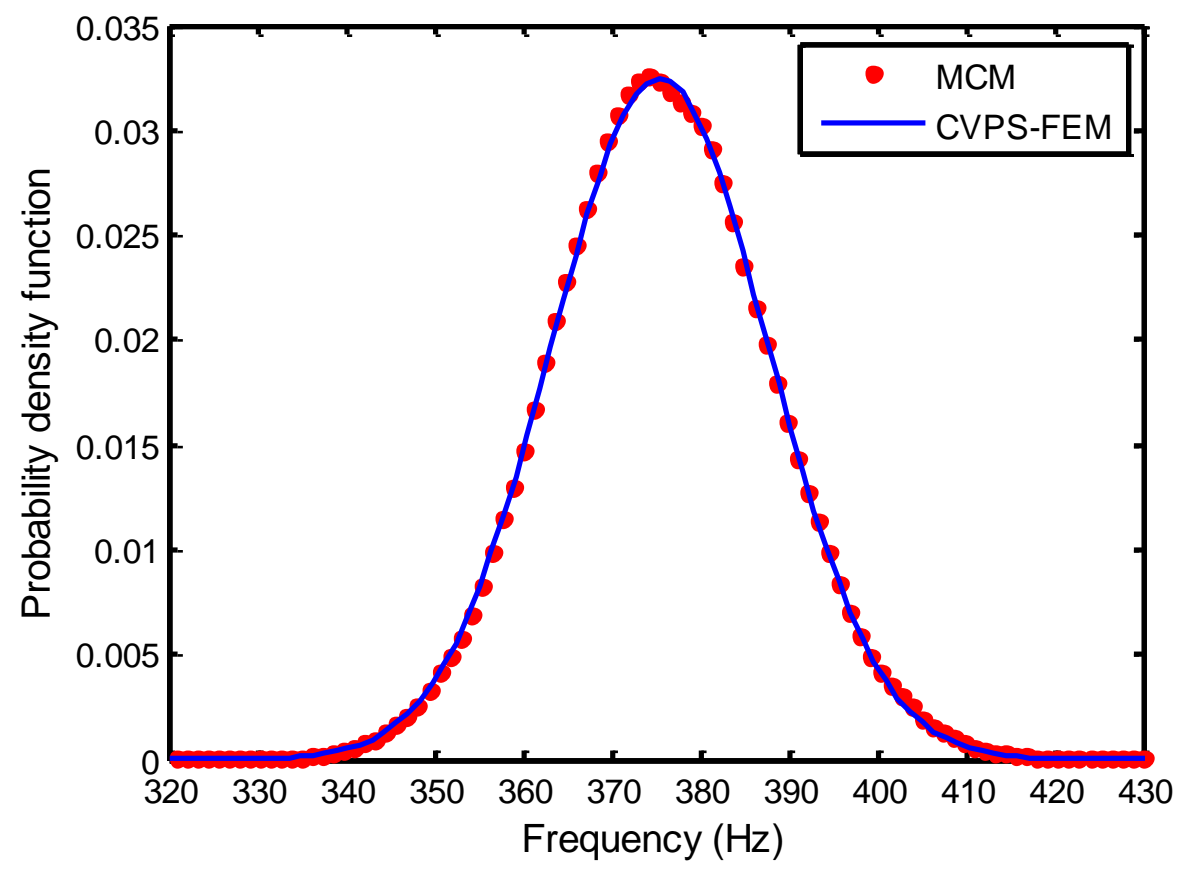

Fig. 11. The probability density function of the $3^{\text {rd }}$ natural frequency along $X$ direction $\left(Q_{2}\right)$ of LRAMs with random parameters. 
Figure 12 shows the $3^{\text {rd }}$ mode shape along X direction of LRAMs unit cell with mean properties. The probability density functions of the normalized $x$-displacement and $y$-displacement of node $N_{2}$ in the $3^{\text {rd }}$ mode shape computed by CVPS-FEM and MCM with considering the random material properties are plotted in Fig. 13. It can be easily observed that an excellent agreement is given between the results obtained by CVPS-FEM and MCM. The robustness and accuracy of the proposed CVPS-FEM is proved clearly in dealing with ternary LRAMs with random variables.

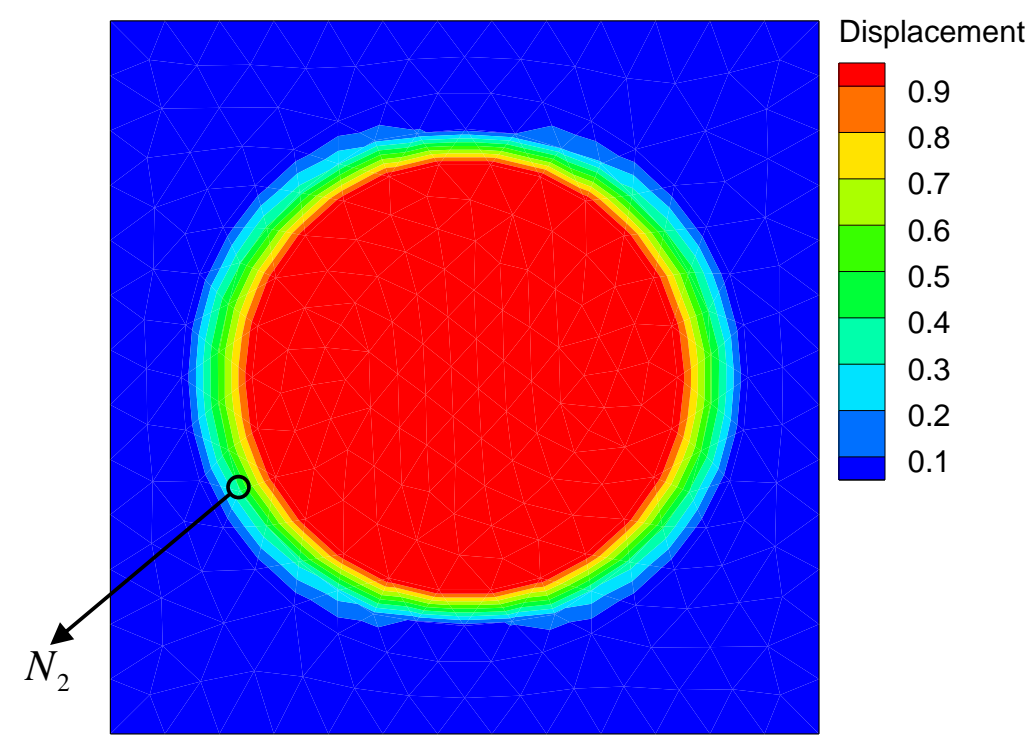

Fig. 12. The $3^{\text {rd }}$ mode shape along $X$ direction of LRAMs unit cell with mean properties. 

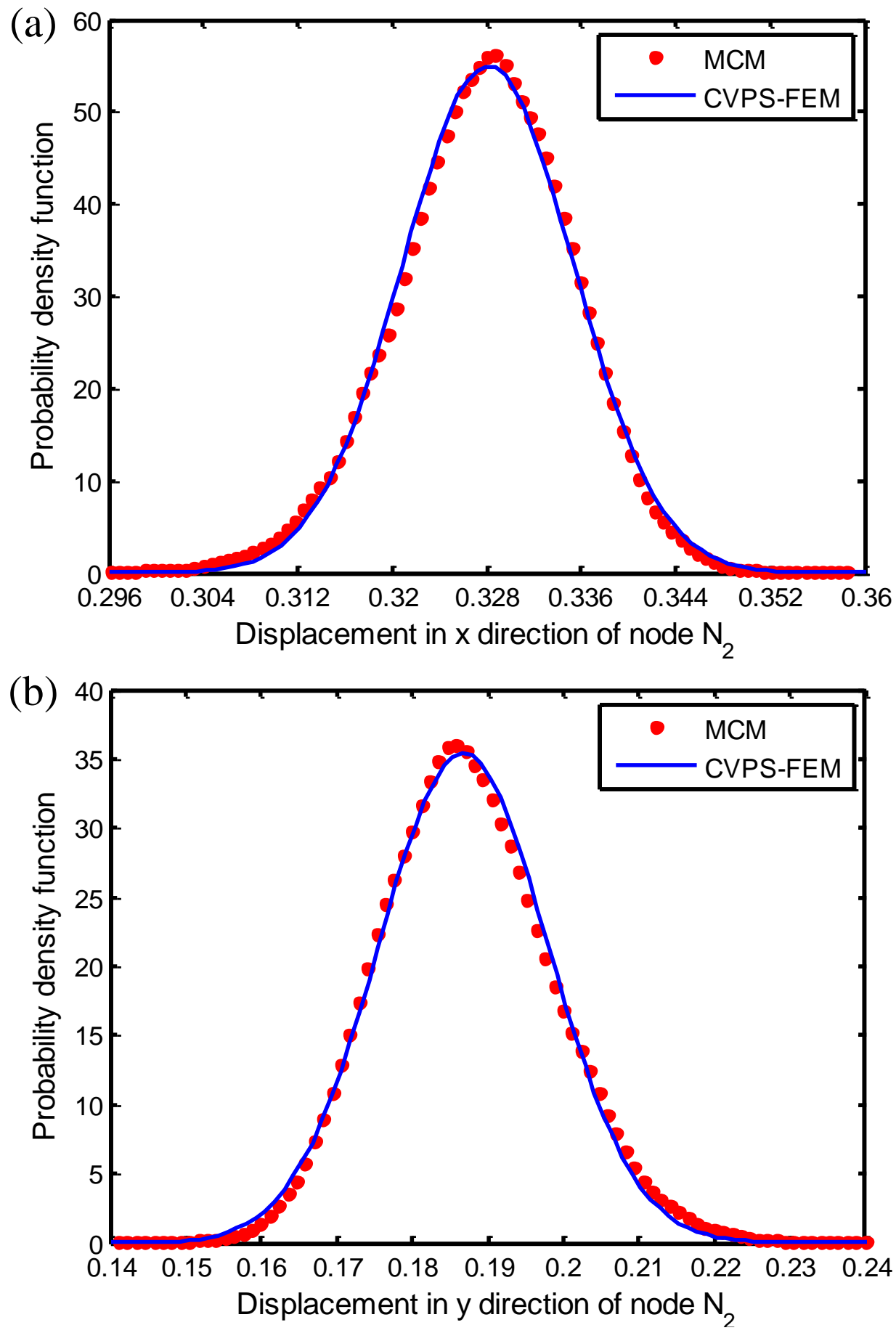

Fig. 13. The probability density function of the normalized nodal displacements in $\mathrm{x}$ and $\mathrm{y}$ direction of node $N_{2}$ of the $3^{\text {rd }}$ mode shape along X direction of LRAMs unit cell with random parameters. (a) The normalized nodal displacement in $x$ direction and (b) the normalized nodal displacement in $y$ direction.

The probability density functions of frequency response of the ternary LRAMs with random parameters at $200 \mathrm{~Hz}$ from CVPS-FEM and MCM are shown in Fig. 14. Again, the 
numerical results for the probability density function of the frequency response using CVPS-FEM match the reference results obtained from MCM very well. It is obviously noticed that the $95 \%$ confidence interval for the response amplitudes is about 1.026 to 1.031 .

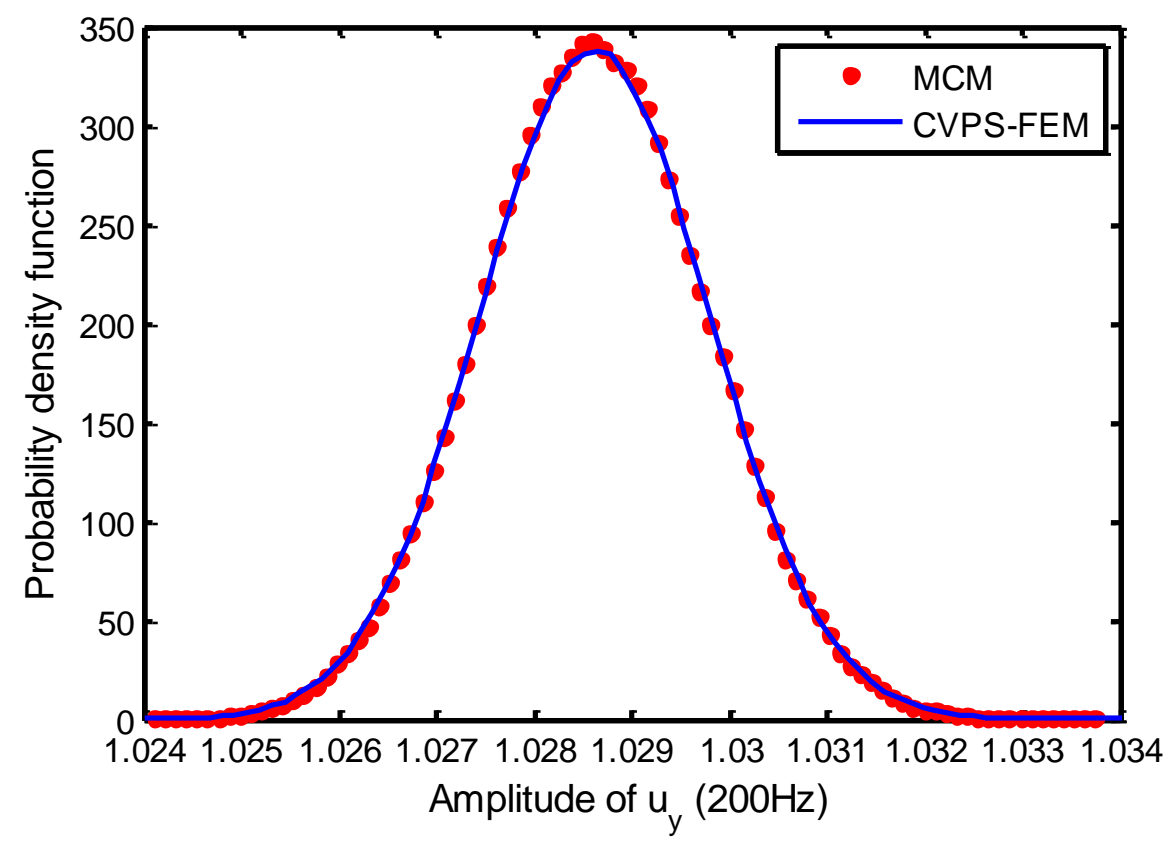

Fig. 14. The probability density function of the frequency response at $200 \mathrm{~Hz}$ of LRAMs with random parameters.

\subsection{Defects in $A M s$}

With the development of AMs, a new type AMs with considering defects has attracted great attention recently as it has great application prospect such as the waveguide field [46]. Unfortunately, due to the manufacturing error, the discrepancy between the design and actual manufacturing of AMs is unavoidable, which leads to the uncertainty of waveguide. Hence, it is of great significance to analyze this type AMs with considering the manufacturing error. As shown in Fig. 15(a), a $5 \times 5$ super-cell AMs with point defect is proposed and investigated in this example. The square AMs structure is made up of epoxy with periodical square-shaped inclusions of lead columns. The lattice constant is $a=0.5 \mathrm{~m}$, 
and the side length of columns lead is $b=0.06 \mathrm{~m}$.

Similarly, in order to calculate the frequency response, the unit forced displacement is applied to the lower side boundary of the $5 \times 5$ super-cells in $y$ direction and the frequency response is obtained at the center of its upper side boundary, which can be seen clearly in Fig. 15(b).
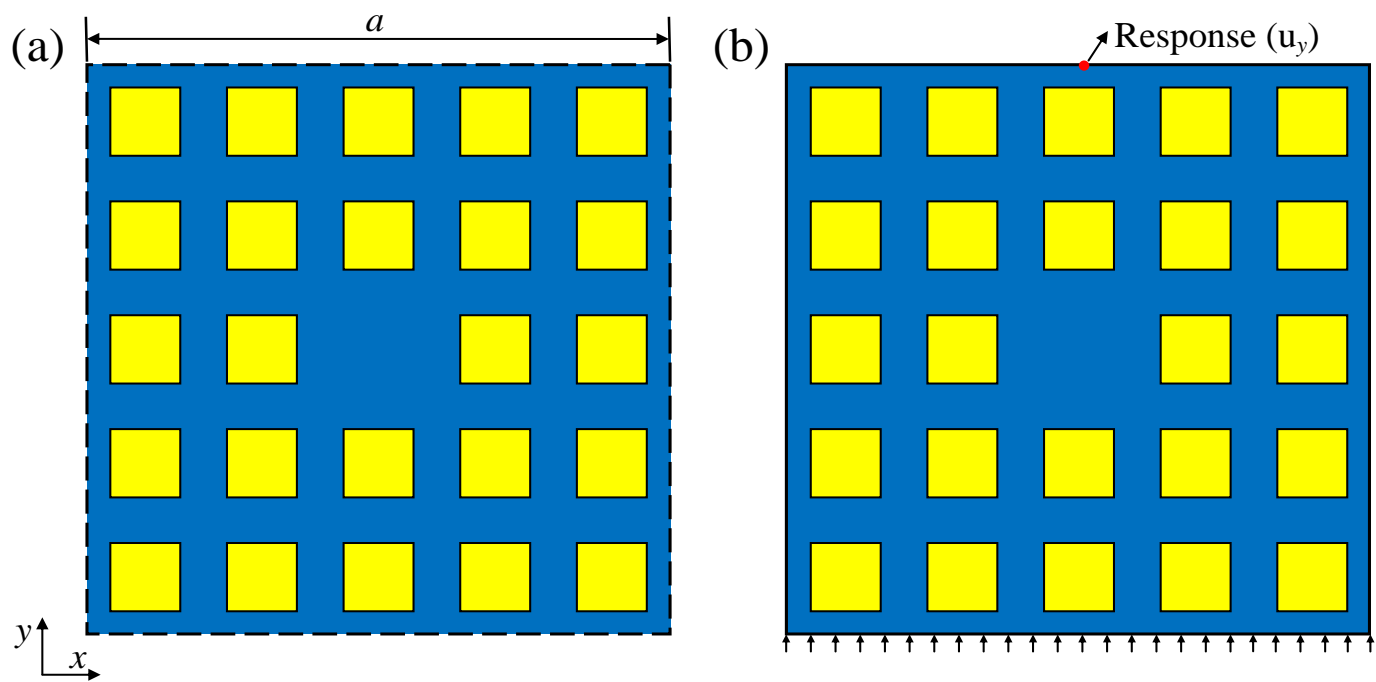

Fig. 15. The $5 \times 5$ super-cells and the response function calculation model of AMs with point defect. (a) The $5 \times 5$ super-cells and (b) the response function calculation model.

The band structure and frequency response function of the AMs with mean properties are calculated as shown in Fig. 16. Similarly, it can be seen clearly from Fig. 16 that the vibration is reduced effectively at the frequencies where the band gaps exist. In addition, because of the existence of the point defect, the defective modes are unavoidable and the frequency responses increase at these frequencies where the defective modes exist. As shown in Fig 16(b), the frequency response increases obviously at the frequency range around $8000 \mathrm{~Hz}$. 

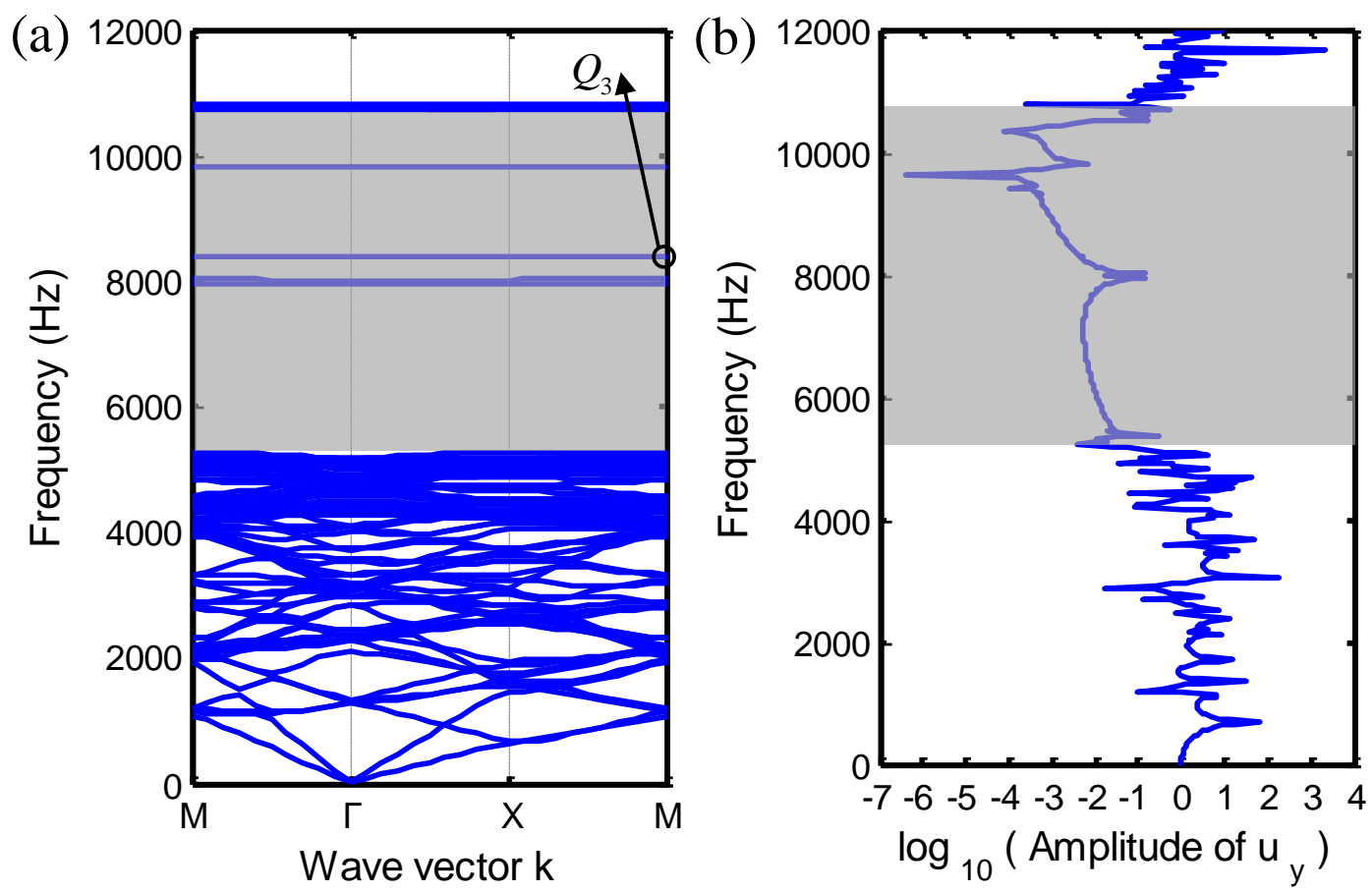

Fig. 16. The band structure and frequency response function of the defective AMs with mean properties. (a)

The band structure and (b) the frequency response function.

Similarly, the material parameters of epoxy and lead are assumed to be random variables as listed in Table 1, and the uncertainty method based on CVPS-FEM is employed to predict the probability density functions of band structure, mode shape and frequency response function of stochastic defective AMs.

Without loss of generality, the uncertain natural frequency of the defective mode in the band structure is analyzed, and the probability density function of the random $75^{\text {th }}$ natural frequency along $\mathrm{M}$ direction (point $Q_{3}$ (corresponding to the defective mode) in Fig. 16(a)) of the AMs with random parameters obtained by CVPS-FEM and MCM is shown in Fig. 17. It can be seen from Fig. 17 that the results computed by CVPS-FEM are very close to the solutions obtained by MCM. It is observed from Fig. 17 that the variance for the natural frequencies is about 11400 by introducing the random parameters, and the $95 \%$ confidence interval for the natural frequencies is about 8180 to 8600 , which leads to the incorrect design 
and application of the AMs.

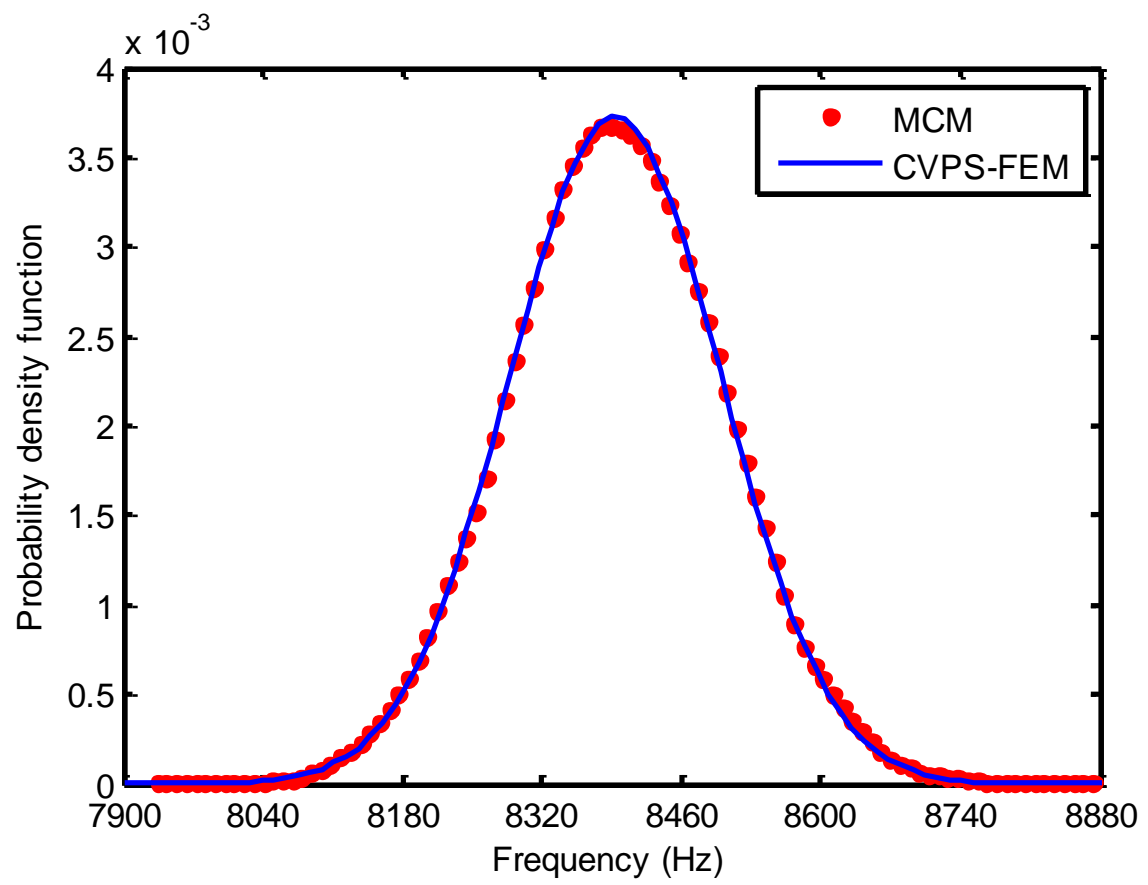

Fig. 17. The probability density function of the $75^{\text {th }}$ natural frequency along $\mathrm{M}$ direction $\left(Q_{3}\right)$ of the defective AMs with random parameters.

The $75^{\text {th }}$ mode shape along $\mathrm{M}$ direction of the defective AMs with mean properties is plotted in Fig. 18. Considering the random material properties, the probability density functions of the normalized $x$-displacement and $y$-displacement of node $N_{3}$ in the $75^{\text {th }}$ mode shape calculated by CVPS-FEM and MCM with are plotted in Fig. 19. It can be seen from Fig. 19 that the probability density function of the random normalized nodal displacement in $x$ and $y$ direction calculated by CVPS-FEM match the reference result perfectly, which indicates that the formulated CVPS-FEM is suitable to predict the mode shapes of the defective AMs with random parameters. It is also noticed that the $95 \%$ confidence interval for the normalized nodal displacements in $\mathrm{x}$ and $\mathrm{y}$ direction are about 0.204 to 0.22 and 0.686 to 0.713 by introducing the random parameters, respectively, which has a great effect on the reliability and robustness of the design of AMs. 


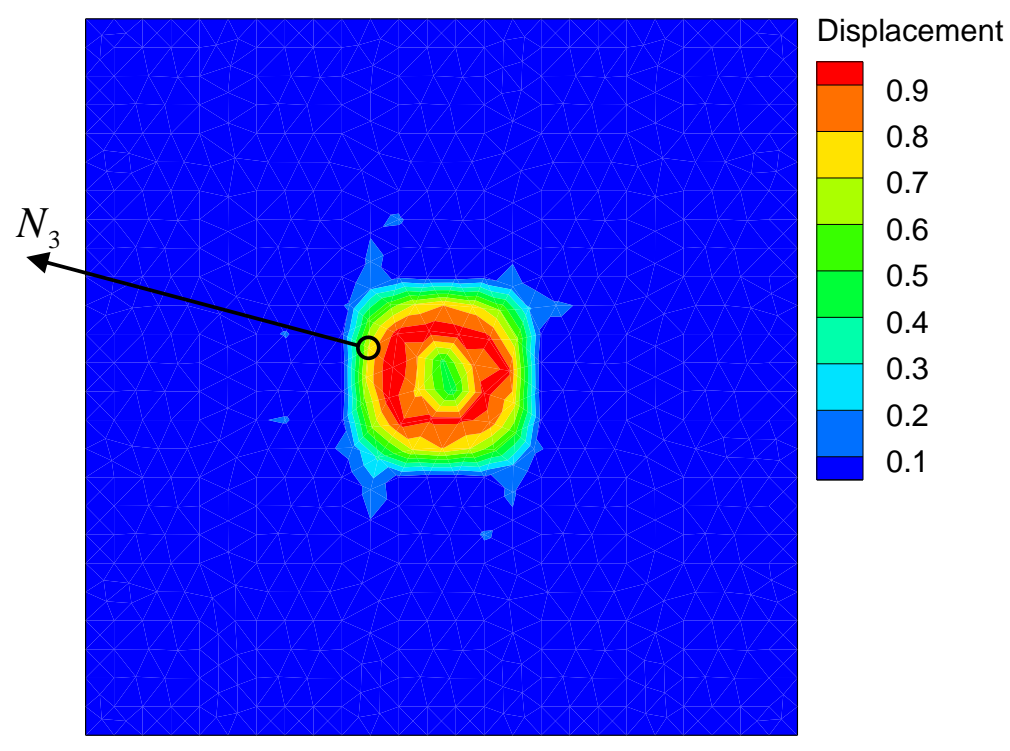

Fig. 18. The $75^{\text {th }}$ mode shape along $M$ direction of the $5 \times 5$ super-cells with mean properties.

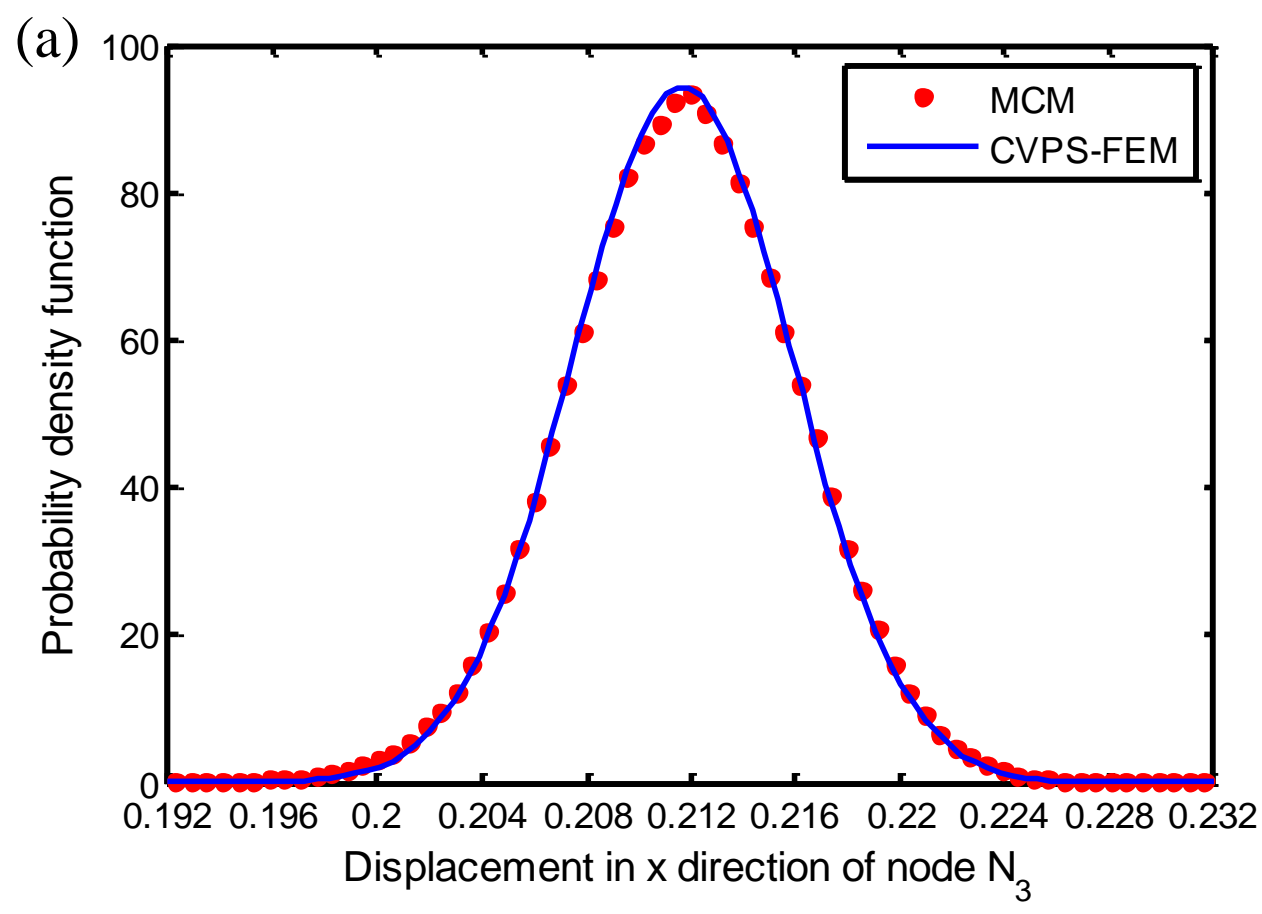




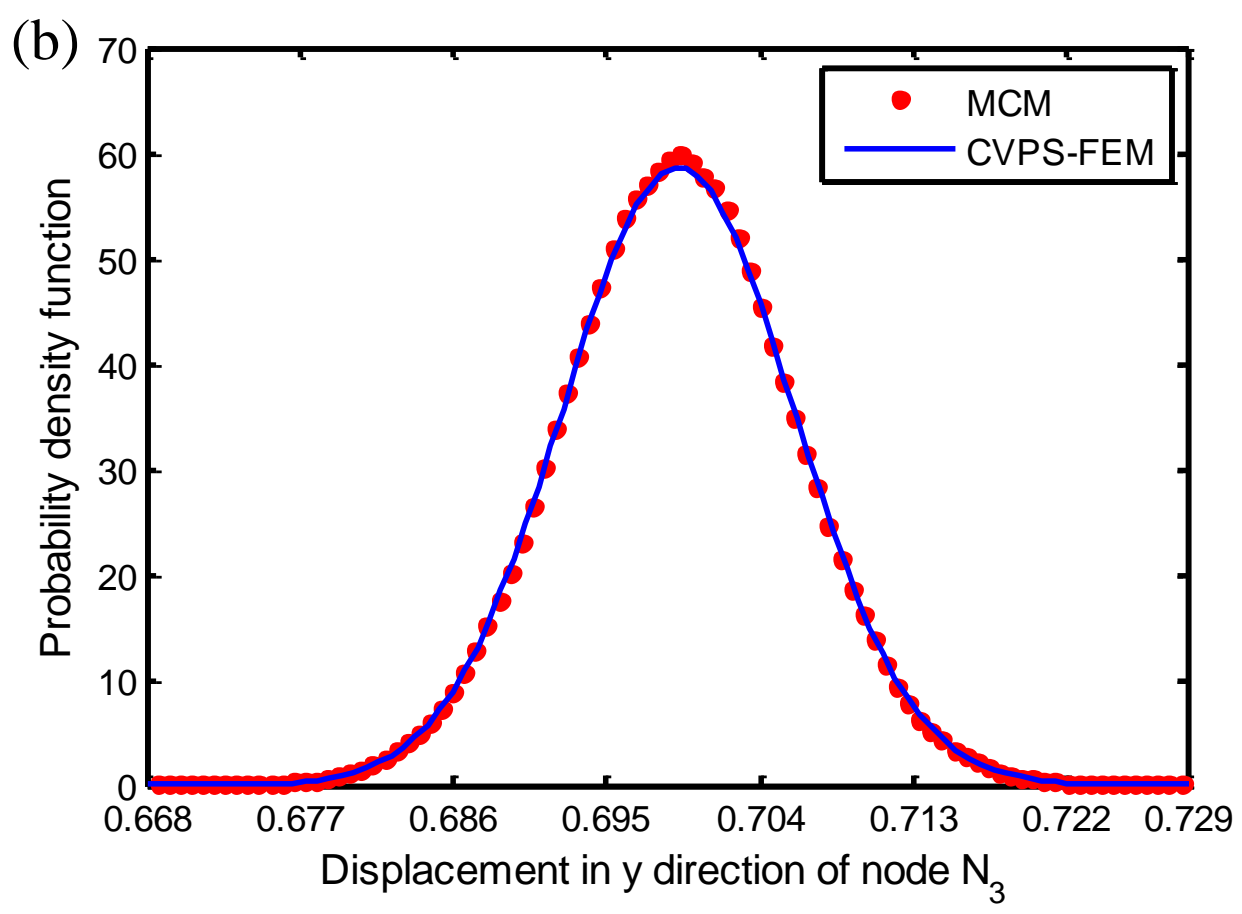

Fig. 19. The probability density function of the normalized nodal displacements in $\mathrm{x}$ and $\mathrm{y}$ direction of node $N_{3}$ of the $75^{\text {th }}$ mode shape along $\mathrm{M}$ direction of the $5 \times 5$ super-cells with random parameters. (a) The normalized nodal displacement in $x$ direction and (b) the normalized nodal displacement in $y$ direction.

The probability density function of the random frequency response at $200 \mathrm{~Hz}$ of the $5 \times 5$ super-cells with random parameters calculated by CVPS-FEM and MCM is shown in Fig. 20. Similarly, it can be seen obviously from Fig. 20 that the numerical results of the probability density function computed by CVPS-FEM are in very good agreement with the solutions obtained by MCM. As shown in Fig. 20, there is a 95\% probability that the nodal displacement is about between 1.108 and 1.124. This example has validated again the effectiveness of the CVPS-FEM in the predication of the probability distribution of the frequency response of the defective AMs with random parameters. 


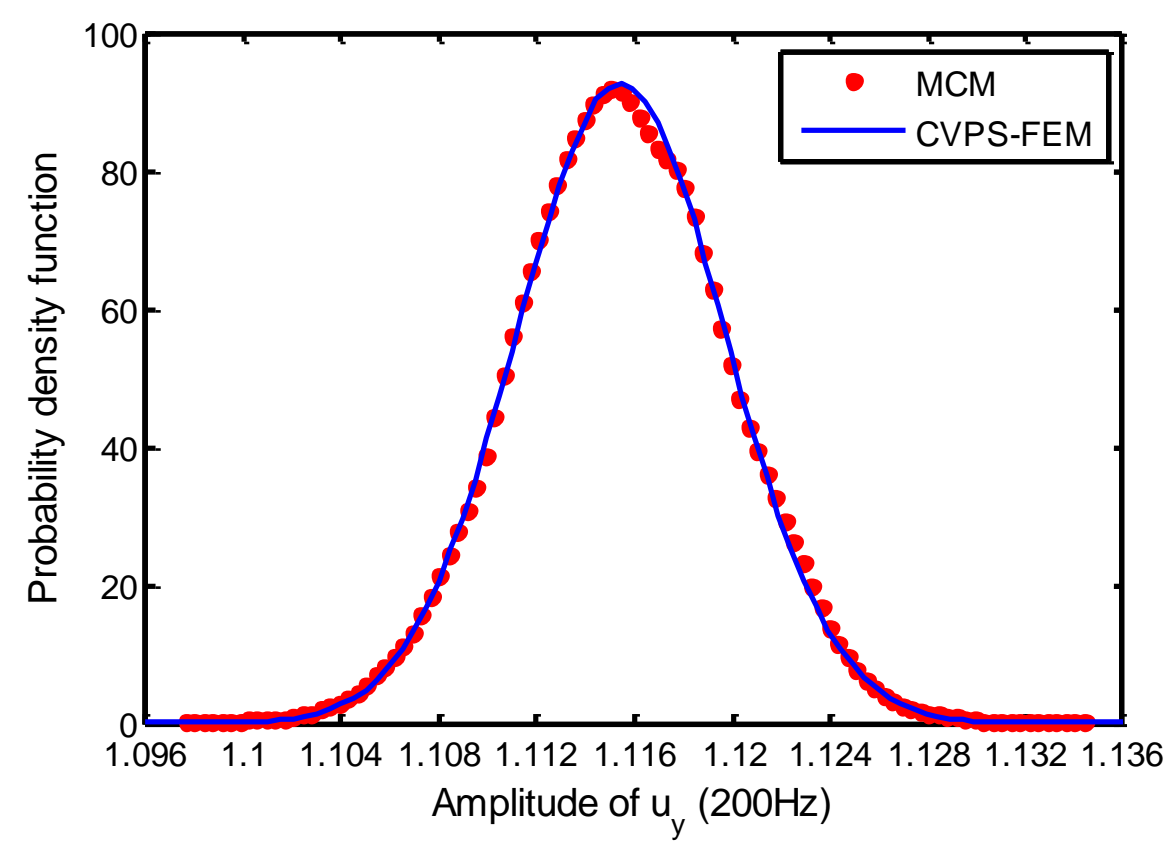

Fig. 20. The probability density function of the frequency response at $200 \mathrm{~Hz}$ of the $5 \times 5$ super-cells with random parameters.

\section{Conclusion}

In order to predict the probability distributions of the physical responses of AMs with random parameters, an efficient uncertainty model based on CVPS-FEM is formulated in this paper. In this uncertainty model, based on the first-order Taylor series expansion and perturbation technique, the physical responses of random AMs can be approximated as linear functions of the random parameters, and then the probability density functions of the responses can be calculated by the change-of-variable technique. The results obtained by MCM are used as the reference results to demonstrate the accuracy of the proposed method. It can be clearly seen from the numerical examples that the CVPS-FEM can predict the band structure, mode shapes and frequency response function of AMs very well compared with MCM. Thus, the uncertainty model based on CVPS-FEM formulated in this paper is very effective to predict the uncertain physical responses of AMs with random parameters, which 
is of great significance for the design and manufacturing of AMs in practical application.

\section{Acknowledgments}

The project is supported by the National Natural Science Foundation of China (Grant No. 51322502) and Project funded by China Postdoctoral Science Foundation. The authors also wish to thank Research Project of State Key Laboratory of Structural Analysis for Industrial Equipment (Grant No. GZ1403), the Science Fund of State Key Laboratory of Advanced Design and Manufacturing for Vehicle Body (Grant No. 51375001), and Research Project of State Key Laboratory of Mechanical Systems and Vibration MSV201613. 


\section{References}

[1] Lu L, Yamamoto T, Otomori M, Yamada T, Izui K, Nishiwaki S. Topology optimization of an acoustic metamaterial with negative bulk modulus using local resonance. Finite Elements in Analysis \& Design. 2013;72:1-12.

[2] Oudich M, Djafari-Rouhani B, Pennec Y, Assouar MB, Bonello B. Negative effective mass density of acoustic metamaterial plate decorated with low frequency resonant pillars. Journal of Applied Physics. 2014;116:377.

[3] Pai PF, Peng H. Acoustic metamaterial structures based on multi-frequency vibration absorbers: International Society for Optics and Photonics, 2014.

[4] Liang Z, Li J. Extreme acoustic metamaterial by coiling up space. Physrevlett. 2012;108:114301.

[5] Krushynska AO, Kouznetsova VG, Geers MGD. Towards optimal design of locally resonant acoustic metamaterials. Journal of the Mechanics \& Physics of Solids. 2014;71:179-96.

[6] Peng H, Pai PF, Peng H. Acoustic metamaterial plates for elastic wave absorption and structural vibration suppression. International Journal of Mechanical Sciences. 2014;89:350-61.

[7] Assouar B, Oudich M, Zhou X. Acoustic metamaterials for sound mitigation. Comptes rendus - Physique. 2016;17:524-32.

[8] Seddon N, Bearpark T. Observation of the inverse Doppler effect. Science. 2003;302:1537-40.

[9] Alu A, Engheta N. Plasmonic and metamaterial cloaking: physical mechanisms and potentials. Journal of Optics A Pure \& Applied Optics. 2008;10:93002-18(17).

[10] Nouh M, Aldraihem O, Baz A. Wave propagation in metamaterial plates with periodic local resonances. Journal of Sound \& Vibration. 2015;341:53-73.

[11] Zhu R, Liu XN, Hu GK, Sun CT, Huang GL. A chiral elastic metamaterial beam for broadband vibration suppression. Journal of Sound \& Vibration. 2014;333:2759-73. 
[12] Zhang S. Acoustic metamaterial design and applications. Dissertations \& Theses Gradworks. 2010.

[13] Zhu R, Huang GL, Huang HH, Sun CT. Experimental and numerical study of guided wave propagation in a thin metamaterial plate. Physics Letters A. 2011;375:2863-7.

[14] Peng H, Pai PF, Deng H. Acoustic Multi-stopband Metamaterial Plates Design for Broadband Elastic Wave Absorption and Vibration Suppression. International Journal of Mechanical Sciences. 2015;103:104-14.

[15] Liu Z, Zhang X, Mao Y, Zhu YY, Yang Z, Chan CT, et al. Locally resonant sonic materials. Science. 2000;289:1734-6.

[16] Sheng P, Zhang XX, Liu Z, Chan CT. Locally resonant sonic materials. Science. 2003;338:201-5.

[17] Jensen JS. Phononic band gaps and vibrations in one- and two-dimensional mass-spring structures. Journal of Sound \& Vibration. 2003;266:1053-78.

[18] Yao S, Zhou X, Hu G. Experimental study on negative effective mass in a 1D mass spring system. New Journal of Physics. 2008;10:043020.

[19] Shi S, Chen C, Prather DW. Plane-wave expansion method for calculating band structure of photonic crystal slabs with perfectly matched layers. Journal of the Optical Society of America A. 2004;21:1769-75.

[20] Hao Y, Mittra R. FDTD modeling of metamaterials. Theory and applications. 2009.

[21] Shen L, He S, Xiao S. A finite-difference eigenvalue algorithm for calculating the band structure of a photonic crystal. Computer Physics Communications. 2002;143:213-21.

[22] Argyropoulos C, Kallos E, Hao Y. FDTD analysis of the optical black hole. Journal of the Optical Society of America B. 2010;27:2020-5.

[23] Wang G, Wen J, Liu Y, Wen X. Lumped-mass method for the study of band structure in two-dimensional phononic crystals. Physical Review B. 2004;69:1324-32.

[24] Yang W, Li J, Huang Y. Modeling and analysis of the optical black hole in

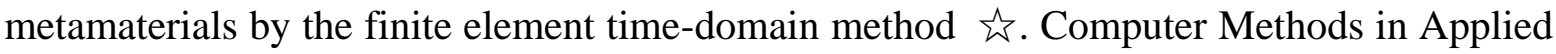
Mechanics \& Engineering. 2016;304:501-20. 
[25] Li J. Error analysis of mixed finite element methods for wave propagation in double negative metamaterials. Journal of Computational \& Applied Mathematics. 2007;209:81-96. [26] He ZC, Li E, Wang G, Li GY, Xia Z. Development of an efficient algorithm to analyze the elastic wave in acoustic metamaterials. Acta Mechanica. 2016:1-16.

[27] Li E, He ZC, Wang G. An exact solution to compute the band gap in phononic crystals. Computational Materials Science. 2016;122:72-85.

[28] Li E, He ZC, Hu JY, Long XY. Volumetric locking issue with uncertainty in the design of locally resonant acoustic metamaterials. Computer Methods in Applied Mechanics \& Engineering. 2017;324.

[29] Li E, He ZC, Wang G, Liu GR. An ultra-accurate numerical method in the design of liquid phononic crystals with hard inclusion. Computational Mechanics. 2017:1-14.

[30] Bessa MA, Bostanabad R, Liu Z, Hu A, Apley DW, Brinson C, et al. A framework for data-driven analysis of materials under uncertainty: Countering the curse of dimensionality. Computer Methods in Applied Mechanics \& Engineering. 2017;320:633-67.

[31] Bostanabad R, Bui AT, Xie W, Apley DW, Chen W. Stochastic microstructure characterization and reconstruction via supervised learning. Acta Materialia. 2016;103:89-102.

[32] Feng, Y.T, Li, C.F, Owen, D. RJ. A directed Monte Carlo solution of linear stochastic algebraic system of equations. Finite Elements in Analysis \& Design. 2010;46:462-73.

[33] Tartakovsky DM, Xiu D. Stochastic analysis of transport in tubes with rough walls. Journal of Computational Physics. 2006;217:248-59.

[34] Ghanem RG, Spanos PD. Stochastic Finite Elements: A Spectral Approach. Springer Berlin. 1991;224.

[35] Wang M, Huang Q. A new hybrid uncertain analysis method for structural-acoustic systems with random and interval parameters. Computers \& Structures. 2016;175:15-28.

[36] Kang Z, Cheng GD. Structural robust design based on perturbation stochastic finite element method. Chinese Journal of Computational Mechanics. 2006;23:129-35. 
[37] Papadimitriou C, Katafygiotis LS, Beck JL. Approximate analysis of response variability of uncertain linear systems. Probabilistic Engineering Mechanics. $1995 ; 10: 251-64$.

[38] Culla A, Carcaterra A. Statistical moments predictions for a moored floating body oscillating in random waves. Journal of Sound \& Vibration. 2007;308:44-66.

[39] Xia B, Yu D, Liu J. Transformed perturbation stochastic finite element method for static response analysis of stochastic structures. Finite Elements in Analysis \& Design. 2014;79:9-21.

[40] LI J, WANG Y, ZHANG C. DISPERSION RELATIONS OF A PERIODIC ARRAY OF FLUID-FILLED HOLES EMBEDDED IN AN ELASTIC SOLID. Journal of Computational Acoustics. 2012;20:237-47.

[41] Liu GR. On Partitions of Unity Property of Nodal Shape Functions: Rigid-Body-Movement Reproduction and Mass Conservation. International Journal of Computational Methods. 2016;13:1640003.

[42] Liu G, Xi Z, Horie Y. Elastic Waves in Anisotropic Laminates. Applied Mechanics Reviews. 2001;56:B23.

[43] Xia B, Yu D. Interval analysis of acoustic field with uncertain-but-bounded parameters. Computers \& Structures. 2012;s 112-113:235-44.

[44] Liu GR. Meshfree Methods: Moving Beyond the Finite Element Method, Second Edition. Crc Press. 2009.

[45] Wang C, Gao W, Song C, Zhang N. Stochastic interval analysis of natural frequency and mode shape of structures with uncertainties. Journal of Sound \& Vibration. 2014;333:2483-503.

[46] Dossou KB, Botten LC, Wilcox S, Mcphedran RC, Sterke CMD, Nicorovici NA, et al. Exact modelling of generalised defect modes in photonic crystal structures. Physica B Condensed Matter. 2007;394:330-4. 